\title{
AMT Starting Control as a Soft Starter Using a Novel Data-Driven Method
}

\section{Yunxia Li ( $\nabla$ ldyffr@126.com )}

a. Qilu University of Technology (Shandong Academy of Sciences), School of Mechanical \& Automotive Engineering, Jinan

\section{Research Article}

Keywords: automated mechanical transmission, data-driven method, model-free adaptive control, jerk compensation, soft start

Posted Date: May 11th, 2021

DOI: https://doi.org/10.21203/rs.3.rs-497927/v1

License: (c) (1) This work is licensed under a Creative Commons Attribution 4.0 International License. Read Full License 


\title{
AMT Starting Control as a Soft Starter Using a Novel Data-Driven Method
}

\author{
Yunxia $\mathrm{Li}^{\mathrm{a}, \mathrm{b}}$ \\ a. Qilu University of Technology (Shandong Academy of Sciences), School of \\ Mechanical \& Automotive Engineering, Jinan, China \\ b. Shandong Institute of Mechanical Design and Research, Jinan, China
}

\begin{abstract}
To improve the starting quality of automated mechanical transmission (AMT) as a soft starter for belt conveyors, a novel data-driven method is proposed. By analyzing the common softstarting acceleration curves, a segmented acceleration curve is proposed as the soft-starting acceleration curve for AMT. A modified model free adaptive control (MFAC) method with jerk compensation is proposed as a data-driven method to control the AMT output shaft's angular acceleration, which consists of a MFAC algorithm and a jerk compensation algorithm. Simulation comparisons are analyzed between the modified MFAC method with jerk compensation, the prototype MFAC method, and the proportion integration differentiation (PID) method. Results indicate better control of the modified MFAC method with jerk compensation on the AMT output shaft's angular acceleration than the other two methods. It also has excellent properties of small tracking error and small shock, providing a novel approach for AMT as a soft starter.
\end{abstract}

Key words: automated mechanical transmission; data-driven method; model-free adaptive control; jerk compensation; soft start

\section{I . Introduction}

In recent decades, the belt conveyors play an important role for the dry bulk material transport. The conveyor belt is composed of rubber, fiber, and metal, with viscoelastic property and complicated dynamic characteristics [1]. As acknowledged, the vibration of the conveyor belt may cause serious accidents such as belt breaking, belt slipping, and belt wear for long-distance and high-power belt conveyors during the starting process [2]-[5]. Therefore, softly starting the belt conveyors is very important. Many scholars have proposed some "S" style speed curves. From the available literatures, five soft-starting acceleration curves exist for belt conveyors including the constant acceleration curve, the sine acceleration curve, the triangle acceleration, the trapezoidal acceleration, and the parabola acceleration [6]-[7]. Reasonable acceleration starting curve can decrease belt vibration and belt accidents.

Currently, the common soft starters of the belt conveyors include the variable frequency and hydro-viscous drives. The principle of the variable frequency drives is to adjust the frequency to change the motor speed [8]. The hydro-viscous drives mainly use the wet clutch to change the speed difference between the active and driven plates [9]-[10]. The variable frequency drives are used in dry locations with sufficient ventilation for the use requirements of the electrical equipment. The hydro-viscous drives are used in cleaning and in airy places for their high-quality oil and high maintenance costs. The above soft starters cannot be widely used under the mine due to high price and high maintenance cost.

AMT is widely used in heavy-duty trucks and proposed to be used as a new soft starter for the belt conveyors with $400 \mathrm{KW}$ in this paper. It has good advantages of low price, low maintenance cost and high efficiency [11]-[12]. It can be used as a new soft starter for belt conveyors through 
gradual shifting as elaborated in the author's published papers [13]-[14]. Figure 1 illustrates the AMT soft-starting system. The AMT soft-starting system includes a three-phase asynchronous motor, an AMT, a reducer, and a belt conveyor. The AMT control system consists of a Transmission Control Unit (TCU), several sensors and several actuators.

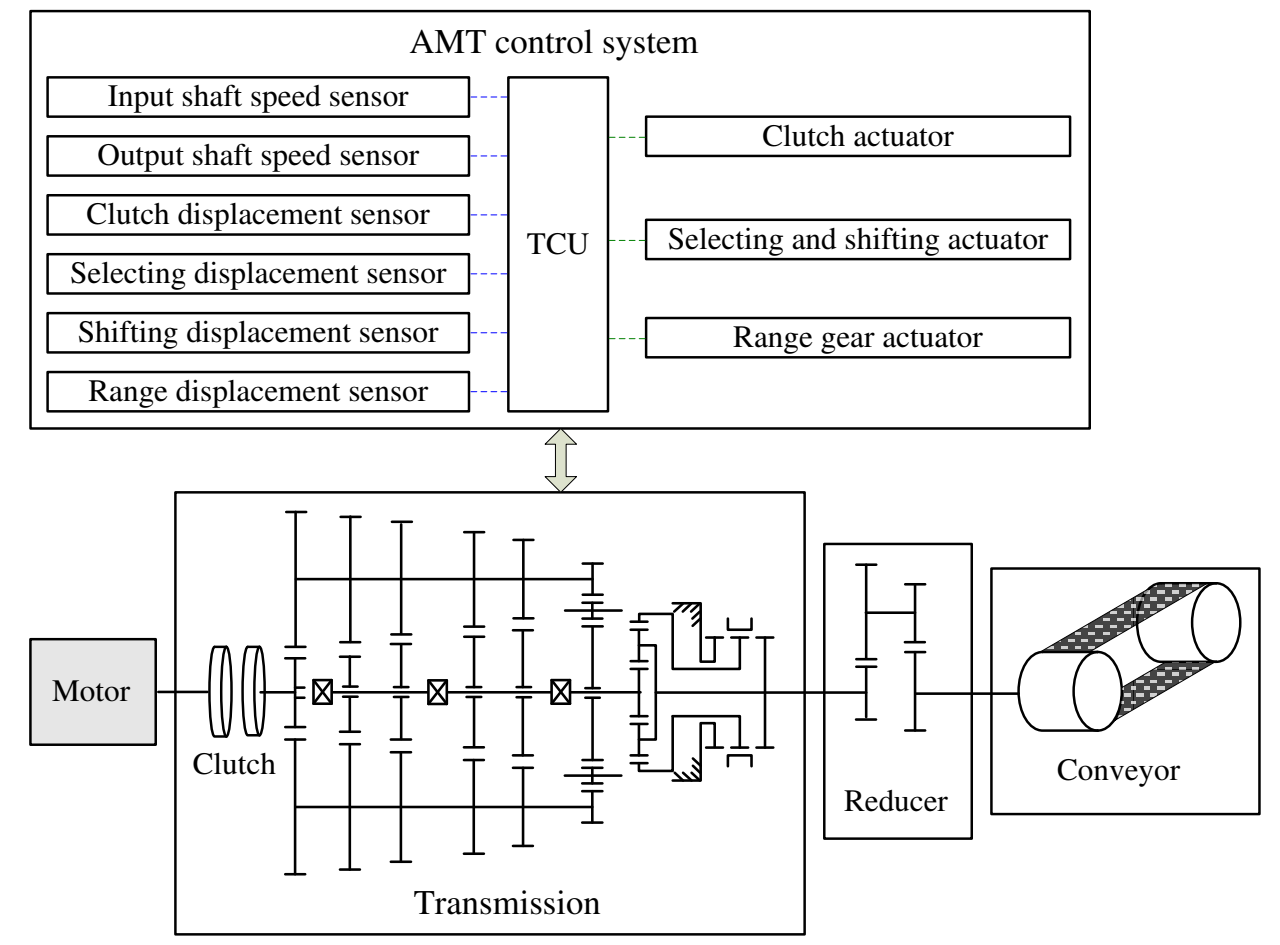

Figure1. Diagram of AMT soft-starting system.

The clutch combines or cuts the transfer of motivity by controlling the clutch engagement and disengagement. In particular, the clutch engagement process includes the empty, resistance, acceleration, and full engagement stages in this paper. Their tasks are eliminating the free clearance, overcoming the running resistance force, controlling the AMT output shaft's angular acceleration, and separately maintaining a running speed under a certain gear position. The key stage is the acceleration stage in which the AMT output shaft's angular acceleration can be controlled and the conveyor belt can be started up softly.

Describing the clutch torque transmissibility is difficult due to the mechanical property of the diaphragm spring and the nonlinear property of the friction disk. The estimation of the clutch transmitting torque has been studied in recent years [15]-[17]. The clutch torque transmissibility can be considered to have nonlinear characteristics. Moreover, the clutch actuator has some hysteresis characteristics. In practice, controlling the AMT output shaft's angular acceleration to accurately track the ideal given curve is difficult.

The speed signal of the AMT output shaft changes slowly if the output shaft speed is lower than $20 \mathrm{rpm}$. Therefore, the control methods based on model are not suitable for the clutch control. Datadriven control methods have better control performances for the complicated and nonlinear actuators, thereby eliminating the need to build an accurate mathematical model. Only depending on the input and output data, data-driven control methods can achieve adaptive control for these complicated and nonlinear actuators. Some data-driven control methods such as PID, fuzzy PID, Active Disturbance Rejection Control (ADRC), Iterative Learning Control (ILC), and MFAC are widely used in industrial control [18]-[21]. Hou proposed the MFAC method which has some better 
control characteristics. In this paper, it is selected as the basic method to better control the AMT output shaft's angular acceleration in meeting the requirement of the belt conveyor's soft starting.

The belt shock will occur if the AMT output shaft's angular jerk exceeds a certain threshold. To avoid a bad situation, the AMT output shaft's angular jerk requires restriction. Then, a modified MFAC method with jerk compensation is proposed as the optimal control method to control the AMT output shaft's angular acceleration for AMT as a soft starter.

\section{Starting acceleration curve based on AMT}

The common starting acceleration expressions are illustrated below.

The constant acceleration curve can be expressed in equation (1).

$$
a(t)=\frac{1}{T_{s}} v_{b} \quad\left(0 \leq t \leq T_{s}\right)
$$

Where $v_{b}$ is the belt target speed, $T_{s}$ is the starting time.

The sine acceleration curve can be given in equation (2).

$$
a(t)=\frac{\pi}{2 T_{s}} v_{b} \sin \left(\frac{\pi}{T_{s}} t\right) \quad\left(0 \leq t \leq T_{s}\right)
$$

The triangular acceleration curve can be written in equation (3).

$$
a(t)= \begin{cases}4 v_{b} \frac{t}{T_{s}^{2}} & \left(0 \leq t \leq T_{s} / 2\right) \\ 4 v_{b}\left(\frac{1}{T_{s}}-\frac{t}{T_{s}^{2}}\right) & \left(T_{s} / 2<t \leq T_{s}\right)\end{cases}
$$

The trapezoidal acceleration curve can be written in equation (4).

$$
a(t)= \begin{cases}\frac{v_{b}}{(N-1) t_{s}^{2}} t & \left(0 \leq t \leq t_{s}\right) \\ \frac{N v_{b}}{(N-1) T_{s}} & \left(t_{s}<t<T_{s}-t_{s}\right) \\ \frac{N^{2} v_{b}}{(N-1) T_{s}^{2}}\left(T_{s}-t\right) & \left(T_{s}-t_{s} \leq t \leq T_{s}\right)\end{cases}
$$

Where $N$ is a natural number greater than $3, t_{s}$ is the time of the ascent or descent acceleration stage, $t_{s}$ is equal to $T_{s}$ divided by $N$.

The parabola acceleration curve can be written in equation (5).

$$
a(t)=6 v_{b}\left(\frac{t}{T_{s}^{2}}-\frac{t^{2}}{T_{s}^{3}}\right) \quad\left(0 \leq t \leq T_{s}\right)
$$

Under the same starting time, Figure 2 presents the schematic of the above five common starting acceleration curves. Table 1 lists the comparisons of the characteristic parameters including the belt velocity, the belt acceleration, and the belt jerk (the differential of the acceleration).

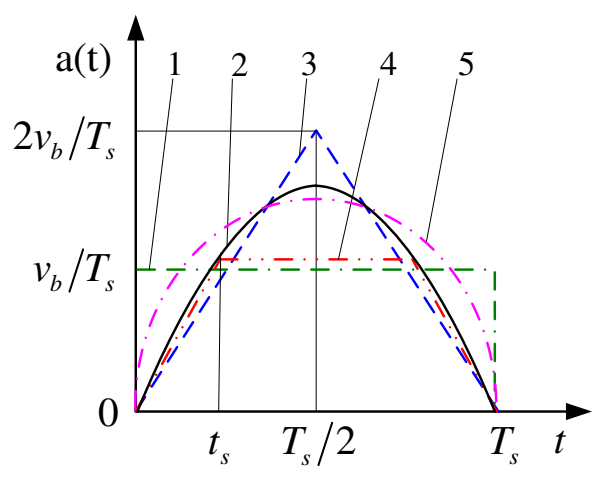


1-costant acceleration curve 2-sine acceleration curve 3-triangular acceleration curve

4- trapezoidal acceleration curve 5- parabola acceleration curve

Figure 2. Five common starting acceleration curves.

Table 1. Comparisons of characteristic parameters for the common starting curves.

\begin{tabular}{|c|c|c|c|c|}
\hline Type & Time & Velocity & Acceleration & Jerk \\
\hline \multirow{2}{*}{$\begin{array}{c}\text { Constant } \\
\text { acceleration }\end{array}$} & 0 & 0 & $v_{b} / T_{s}$ & $\infty$ \\
\hline & $T_{S}$ & $v_{b}$ & $v_{b} / T_{s}$ & $-\infty$ \\
\hline \multirow{3}{*}{$\begin{array}{c}\text { Sine } \\
\text { acceleration }\end{array}$} & 0 & 0 & 0 & $\pi^{2} v_{b} /\left(2 T_{s}^{2}\right)$ \\
\hline & $T_{s} / 2$ & $v_{b} / 2$ & $\pi v_{b} /\left(2 T_{s}\right)$ & 0 \\
\hline & $T_{s}$ & $v_{b}$ & 0 & $-\pi^{2} v_{b} /\left(2 T_{s}^{2}\right)$ \\
\hline \multirow{4}{*}{$\begin{array}{l}\text { Triangular } \\
\text { acceleration }\end{array}$} & 0 & 0 & 0 & $4 v_{b} / T_{s}^{2}$ \\
\hline & $T_{s} / 2$ & $v_{b} / 2$ & $2 v_{b} / T_{s}$ & $4 v_{b} / T_{s}^{2}$ \\
\hline & $T_{s}$ & $v_{b}$ & 0 & $-4 v_{b} / T_{s}^{2}$ \\
\hline & 0 & 0 & 0 & $\frac{N^{2} v_{b}}{(N-1) T_{s}^{2}}$ \\
\hline \multirow{4}{*}{$\begin{array}{l}\text { Trapezoidal } \\
\text { acceleration }\end{array}$} & $T_{s}$ & $v_{b}$ & $N v_{b}$ & $N^{2} v_{b}$ \\
\hline & $\bar{N}$ & $2(N-1)$ & $\overline{(N-1) T_{s}}$ & $\overline{(N-1) T_{s}^{2}}$ \\
\hline & $\frac{(N-1) T_{s}}{N}$ & $\frac{(2 N-3) v_{b}}{2(N-1)}$ & $\frac{N v_{b}}{(N-1) T_{s}}$ & 0 \\
\hline & $T_{S}$ & $v_{b}$ & 0 & $\frac{N^{2} v_{b}}{(N-1) T_{s}^{2}}$ \\
\hline \multirow{3}{*}{$\begin{array}{c}\text { Parabola } \\
\text { acceleration }\end{array}$} & 0 & 0 & 0 & $6 v_{b} / T_{s}^{2}$ \\
\hline & $T_{s} / 2$ & $v_{b} / 2$ & $3 v_{b} /\left(2 T_{s}\right)$ & 0 \\
\hline & $T_{s}$ & $v_{b}$ & 0 & $-6 v_{b} / T_{s}^{2}$ \\
\hline
\end{tabular}

From the perspective of peak acceleration, those of the triangular, sine, and constant acceleration curves occupy the first, second, and last places, respectively. From the perspective of peak jerk, those of the triangular, sine, and constant acceleration curves are described as the lowest, the smaller, and the greatest, respectively. The greater the peak jerk is, the greater the belt vibration. Therefore, the sine and triangular acceleration curve are generally chosen for soft starters.

To meet the requirements of the belt conveyor's soft starting, studying which acceleration curve is more suitable for AMT is necessary. To reduce the clutch wear, reducing the starting times is reasonable. The acceleration algorithm should not be excessively complicated due to some hysteresis properties of the AMT actuators. Following the above analysis, the triangular and trapezoidal acceleration curves are practicable in reducing the starting time and the peak jerk. The belt acceleration can be achieved from zero to its maximum by controlling the duty ratios of the clutch engaging solenoid valve, and it can be accomplished from its maximum to zero by controlling the duty ratios of the clutch disengaging solenoid valve. Combing the above analysis, a segmented acceleration curve is proposed to control the acceleration process for AMT as a soft starter as shown in Figure 3. 


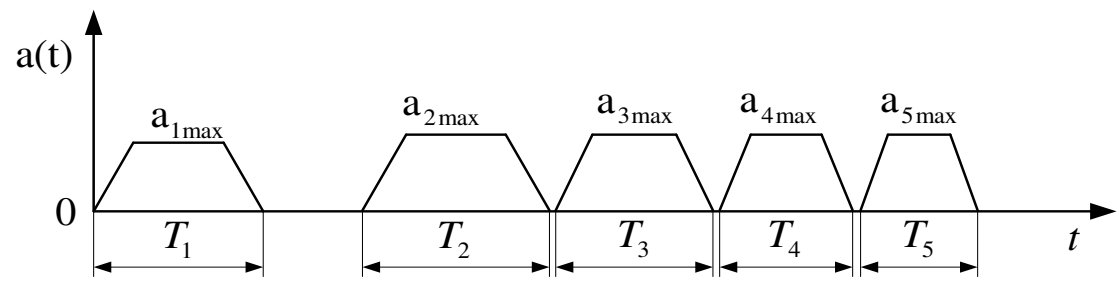

Figure 3. Starting acceleration curve based on AMT.

The segmented acceleration curve can be illustrated as follows. It comprises several trapezoidal acceleration curves determined by the belt speed and gear positions. The first stage of the conveyor's starting process is to accelerate the belt from zero to the target speed under the first gear position which is labeled $T_{1}$ and kept running at its target speed for longer than $5 \mathrm{~s}$ to stretch the entire belt. Then, the clutch should be disengaged and the gear position shifted to the second gear position. The second stage of the conveyor's starting process is to still accelerate the belt from the previous speed to the target speed under the second gear position which is labeled $T_{2}$ and kept running at its speed for $2 \mathrm{~s}$. Then, the clutch should be disengaged again and the gear position shifted to the third gear position. The third stage of the conveyor's starting process is still to accelerate which is labeled $T_{3}$ and kept running at its speed for $2 \mathrm{~s}$. Similarly, the other stages are completed through upshifting until the target belt speed is reached. Finally, the conveyor's soft starting process is completed.

\section{AMT dynamics analysis}

\section{A. Clutch torque transmissibility}

The clutch transmitting torque can be expressed as a third-order polynomial concerning the big end displacement of the diaphragm spring [22]. The clutch actuator is an air cylinder controlled by the solenoid valves, and the piston displacement is proportional to the big end displacement of the diaphragm spring. Therefore, the clutch transmitting torque can also be expressed as a third-order polynomial concerning the clutch actuator displacement. Normally, the clutch friction disk is often worn which will affect the clutch torque transmissibility. Therefore, the clutch transmitting torque can be expressed in equation (6).

$$
T_{c}(x)=\mu^{\prime}\left[K_{1}\left(x-x_{0}-\Delta x_{0}\right)+K_{2}\left(x-x_{0}-\Delta x_{0}\right)^{2}+K_{3}\left(x-x_{0}-\Delta x_{0}\right)^{3}\right]
$$

Where $\mu^{\prime}$ is the friction coefficient of the clutch disk, $x$ is the clutch actuator displacement, $x_{0}$ is the clutch actuator displacement at the critical point of the empty stroke for the new clutch, $\Delta x_{0}$ is the displacement increment of the clutch's empty stroke due to wearing.

\section{B. Transmission dynamic model}

The input and output shafts can be regarded as two mass units. Thus, the transmission model can be built as a double-mass model as shown in Figure 4. 


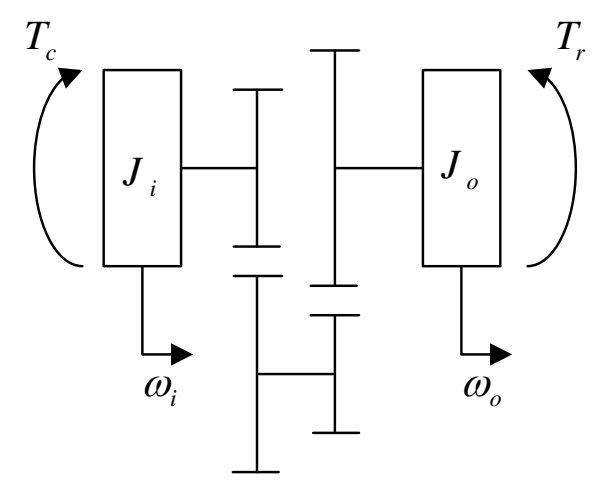

Figure 4. Transmission double-mass model.

Assuming the system damping is ignored, the transmission dynamic equation can be expressed in equation (7).

$$
\left\{\begin{array}{l}
\dot{\omega}_{o}=\frac{T_{c}(x) i_{g} i_{g}-T_{r}}{J_{i} i_{g}^{2}+J_{o}} \\
\ddot{\omega}_{o}=\frac{d \dot{\omega}_{o}}{d t} \\
T_{c}(x)=\mu^{\prime}\left[K_{1}\left(x-x_{0}-\Delta x_{0}\right)+K_{2}\left(x-x_{0}-\Delta x_{0}\right)^{2}+K_{3}\left(x-x_{0}-\Delta x_{0}\right)^{3}\right] \\
T_{r}=\frac{F_{f} r_{d}}{i_{r} \eta_{r}} \\
J_{o}=J_{t}+\frac{J_{c}+J_{r}}{i_{r}^{2}}
\end{array}\right.
$$

(7)

Where $J_{i}, J_{t}, J_{c}$, and $J_{r}$ are the moment of inertia about the AMT input shaft, AMT output shaft, belt conveyor, reducer's output shaft, respectively. $J_{o}$ is the equivalent moment of inertia about the AMT output shaft. $\omega_{i}$ and $\omega_{o}$ are the input and output shaft's angular speeds, respectively. $T_{c}$ and $T_{r}$ are the transmitted torque of the clutch and the equivalent resistance torque of the AMT output shaft, respectively. $F_{f}$ is the running resistance force of the belt conveyor. $r_{d}$ is the head roller's radius of the belt conveyor. $i_{g}$ and $i_{r}$ are the transmission ratio and the reducer ratio. $\eta_{g}$ and $\eta_{r}$ are the AMT efficiency and the reducer efficiency, respectively.

If the sampling interval is $T$, the discrete model of equation (7) can be written in equation (8).

$$
\left\{\begin{array}{l}
\omega_{o}(k+1)=\omega_{o}(k)+T \frac{T_{c}(x(k)) i_{g} \eta_{g}-T_{r}(k)}{\left(J_{i} i_{g}^{2}+J_{o}(k)\right)} \\
\ddot{\omega}_{o}(k)=\frac{\dot{\omega}_{o}(k)-\dot{\omega}_{o}(k-1)}{T} \\
T_{c}(x(k))=\mu^{\prime}\left[K_{1}\left(x(k)-x_{0}-\Delta x_{0}\right)+K_{2}\left(x(k)-x_{0}-\Delta x_{0}\right)^{2}+K_{3}\left(x(k)-x_{0}-\Delta x_{0}\right)^{3}\right] \\
T_{r}(k)=\frac{F_{f(k)} r_{d}}{i_{r} \eta_{r}} \\
J_{o}(k)=J_{t}+\frac{J_{c}(k)+J_{r}}{i_{r}^{2}}
\end{array}\right.
$$

Where $k$ is a natural number. $\omega_{o}(k)$ and $\omega_{o}(k+1)$ are the angular speed at No. $k$ sampling time and that No. $(k+1)$ sampling time separately.

\section{Angular acceleration curve of the AMT output shaft}

The equivalent angular acceleration curve of the AMT output shaft can be given in equation (9) according to equation (4). 


$$
\dot{\omega}_{o}(t)= \begin{cases}\frac{i_{r} v_{b}}{r_{d}(N-1) t_{s}^{2}} t & \left(0 \leq t \leq t_{s}\right) \\ \frac{i_{r} N v_{b}}{r_{d}(N-1) T_{s}} & \left(t_{s} \leq t \leq T_{s}-t_{s}\right) \\ \frac{i_{r} N^{2} v_{b}}{r_{d}(N-1) T_{s}^{2}}\left(T_{s}-t\right) & \left(T_{s}-t_{s} \leq t \leq T_{s}\right)\end{cases}
$$

\section{Acceleration control method}

The ascent stage of the trapezoidal acceleration curve can be achieved by controlling the duty ratio of the engaging solenoid and closing the disengaging solenoid valve. Moreover, the descent stage of the trapezoidal acceleration curve can be accomplished by controlling the duty ratio of the disengaging solenoid valve and closing the engaging solenoid valve. The horizontal stage of the trapezoidal acceleration curve can be accomplished by closing the engaging and disengaging solenoid valves. Based on the prototype MFAC method, the modified MFAC method with jerk compensation is proposed as follows: it is used not only to control the AMT output shaft's angular acceleration but also to reduce the potential shocks during AMT's starting and shifting.

\section{A. Prototype MFAC method}

Figure 5 presents the block diagram of the prototype MFAC method. Given by the designed angular acceleration curve of the AMT output shaft, the MFAC controller controls the duty ratio $u$ of the clutch solenoid valve to control the clutch displacement. Moreover, the AMT output shaft's angular acceleration $\dot{\omega}_{o}$ can be regulated to trace the ideal angular acceleration $\dot{\omega}_{o}{ }^{*}$.

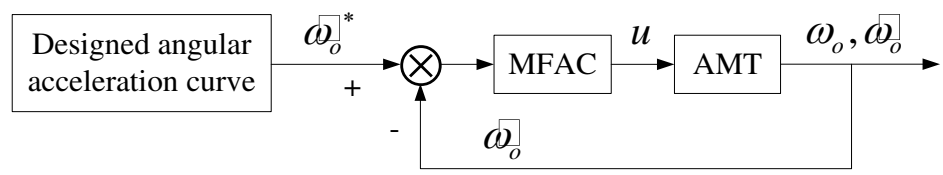

Figure 5. Block diagram of the prototype MFAC method.

The discrete algorithm of the AMT output shaft's angular acceleration can be expressed in equation (10).

$$
\dot{\omega}_{o}(k+1)=\frac{1}{T}\left(\omega_{o}(k+1)-\omega_{o}(k)\right)
$$

Where $\dot{\omega}_{o}(k+1)$ is the AMT output shaft's angular acceleration at No. $(k+1)$ sampling time.

The AMT system can be regarded as a dynamic linear model using the MFAC theory which is driven by data in equation (11).

$$
\left\{\begin{array}{l}
\Delta \dot{\omega}_{o}(k+1)=\phi_{c}(k) \Delta u(k) \\
\Delta \dot{\omega}_{o}(k+1)=\dot{\omega}_{o}(k+1)-\dot{\omega}_{o}(k) \\
\Delta u(k)=u(k)-u(k-1)
\end{array}\right.
$$

Where $\phi_{c}(k)$ is the Pseudo Partial Derivative (PPD) of the system, $\dot{\omega}_{o}(k)$ is the AMT output shaft's angular acceleration at No. $k$ sampling time, $\Delta \omega_{o}(k+1)$ is the increment of the AMT output shaft's angular acceleration at No. $(k+1)$ sampling time, $u(k)$ and $u(k-1)$ are the duty ratios of the solenoid valve at No. $k$ sampling time and that at No. $(k-1)$ sampling time separately.

The weighting square sum based on the forward prediction errors is used as the criterion function to control the input parameter in equation (12).

$$
J(u(k))=\left(\dot{\omega}_{o}^{*}(k+1)-\dot{\omega}_{o}(k+1)\right)^{2}+\lambda(u(k)-u(k-1))^{2}
$$

Where $\lambda$ is a weighting factor which is more than zero to limit the input parameter $u(k)$, 
$\dot{\omega}_{o}{ }^{*}(k+1)$ is the ideal angular acceleration given by the designed angular acceleration curve at No. $(k+1)$ sampling time.

Taking the derivative of equation (12) with respect to $u(k)$ and letting its value be equal to zero, the control algorithm of $u(k)$ is obtained in equation (13).

$$
u(k)=u(k-1)+\frac{\rho \phi_{c}(k)\left(\dot{\omega}_{o}^{*}(k+1)-\dot{\omega}_{o}(k)\right)}{\lambda+\phi_{c}^{2}(k)}
$$

Where $\rho$ is a step factor which is more than zero and less than or equal to 1 to adjust the increment of $\Delta u(k)$.

The estimation criterion function about $\phi_{c}(k)$ can be expressed in equation (14).

$$
J\left(\phi_{c}(k)\right)=\left(\dot{\omega}_{o}(k)-\dot{\omega}_{o}(k-1)-\phi_{c}(k) \Delta u(k-1)\right)^{2}+\mu\left(\phi_{c}(k)-\hat{\phi}_{c}(k-1)\right)^{2}
$$

Where $\mu$ is a penalty factor which is more than zero to limit the changes of $\phi_{c}(k)$.

Taking the derivative of equation (14) with respect to $\phi_{c}(k)$ and letting its value be equal to zero, which means to minimize the estimation criterion function expressed by equation (14), then the estimation algorithm of $\hat{\phi}_{c}(k)$ is obtained in equation (15).

$$
\left\{\begin{array}{l}
\hat{\phi}_{c}(k)=\hat{\phi}_{c}(k-1)+\frac{\eta \Delta u(k-1)}{\mu+(\Delta u(k-1))^{2}}\left(\Delta \dot{\omega}_{o}(k)-\hat{\phi}_{c}(k-1) \Delta u(k-1)\right) \\
\Delta \widehat{\phi}_{c}(k)=\hat{\phi}_{c}(k)-\hat{\phi}_{c}(k-1)
\end{array}\right.
$$

Where $\eta$ is a step factor which is more than zero and less than or equal to 1 to adjust the increment of $\Delta \hat{\phi}_{c}(k)$.

To make the parameters estimation algorithm achieve a good time-tracking feature, the relevant parameters are reset in equation (16) below.

$$
\left\{\begin{array}{l}
\hat{\phi}_{c}(k)=\hat{\phi}_{c}(1) \\
\text { if }\left|\hat{\phi}_{c}(k)\right| \leq \varepsilon \\
\text { or }|\Delta u(k-1)| \leq \varepsilon \\
\text { or } \operatorname{sign}\left(\hat{\phi}_{c}(k)\right) \neq \operatorname{sign}\left(\hat{\phi}_{c}(1)\right)
\end{array}\right.
$$

Where $\hat{\phi}_{c}(1)$ is an initial value of $\hat{\phi}_{c}(k), \varepsilon$ is a very small positive number which provides the flexibility for $\hat{\phi}_{c}(k)$ and $\Delta u(k-1)$.

If $\Delta u(k-1)$ equals zero at No. $k$-1 sampling time, the time should be moved backward until $u(k-1)$ is not equal to $u(k-m)$ at No. $(k-m)$ sampling time. Therefore, the AMT system can be generally transformed into a dynamic linear model based on equation (15).

In conclusion, the algorithm of the prototype MFAC method comprises equations (13), (15), and (16).

\section{B. MFAC method with jerk compensation}

The AMT output shaft's angular jerk is defined as the change in the AMT output shaft's angular acceleration per unit time. The jerk compensation is used to improve the starting quality if the absolute value of the jerk exceeds the set threshold. Figure 6 presents the block diagram of the modified MFAC method with jerk compensation.

The jerk compensation algorithm can be described in equation (17).

$$
\widetilde{\omega}_{o}(k+1)=\dot{\omega}_{o}^{*}(k+1) \pm \xi \frac{\left(\left|\ddot{\omega}_{o}(k)\right|-\ddot{\omega}_{\text {omax }}\right)}{\ddot{\omega}_{\text {omax }}} \dot{\omega}_{o}^{*}(k+1)
$$

Where $\xi$ is a weighting factor which is greater than zero and less than $1, \widetilde{\omega}_{o}(k+1)$ is the modified ideal angular acceleration of the AMT output shaft at No. $k$ sampling time, $\left|\ddot{\omega}_{o}(k)\right|$ is the 
absolute value of the AMT output shaft's angular jerk at No. $k$ sampling time, $\ddot{\omega}_{\text {omax }}$ is the set threshold. "-" and "+" are used during the ascent and descent acceleration stages, respectively.

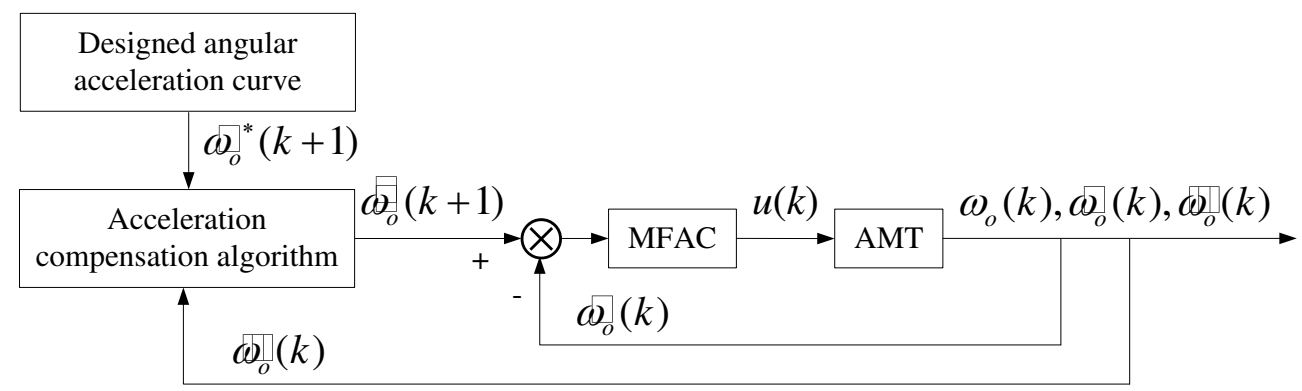

Figure 6. Block diagram of the MFAC method with jerk compensation.

Now, the algorithm of the modified MFAC method with jerk compensation for AMT is given including equations (13), (15), (16), and (17).

\section{Simulation and analysis}

To prove whether the modified MFAC method with jerk compensation is efficient for soft starting, the simulation experiments are thoroughly studied under the first gear position and compared with the prototype MFAC method, the modified MFAC method with jerk compensation, and the PID method.

\section{A. Parameters description}

The basic parameters of the driveline are described below. The power of the three-phase asynchronous motor is $300 \mathrm{KW}$. The driven roller radius of the belt conveyor is $0.5 \mathrm{~m}$. The motor rated speed is $1,480 \mathrm{rpm}$. The belt conveyor's equivalent running mass and equivalent running resistance force with full load are $248,696 \mathrm{~kg}$ and $50,819 \mathrm{~N}$, respectively. The first gear ratio of AMT is 14.28 . The transmission efficiency of AMT is approximately 0.96 . The equivalent moment of inertia about the AMT output shaft is $10 \mathrm{~kg} \cdot \mathrm{m}^{2}$. The moment of inertia about the clutch active parts (the pressure plate and the flywheel) and that of the clutch disk are $1.3 \mathrm{~kg} \cdot \mathrm{m}^{2}$ and 0.135 $\mathrm{kg} \cdot \mathrm{m}^{2}$, respectively. The transmission efficiency of the reducer is approximately 0.97 .

If $x_{d}$ is the big end displacement of the diaphragm spring, the transmitted torque of the clutch is a function about the big end displacement of the diaphragm spring in the literature [23] which can be expressed in equation (18).

$$
T_{c}=\mu^{\prime}\left(3464.483459 x_{d}-336.521878 x_{d}{ }^{2}+7.775271 x_{d}{ }^{3}\right)
$$

If the friction coefficient $\mu^{\prime}$ equals 0.25 , the lever ratio between the clutch actuator displacement and the big end displacement of the diaphragm spring is 6 , the value of $x_{0}$ is $10 \mathrm{~mm}$, and $x(k)$ is the clutch actuator displacement at No. $k$ sampling time. Then, the discrete model of the transmitted torque of the new clutch can be expressed in equation (19) according to equation (6). $T_{c}(x(k))=144.353477(x(k)-10)-2.336957(x(k)-10)^{2}+0.008999(x(k)-10)^{3}$

The engaging solenoid valve can be fully closed if the duty ratio is less than 0.2 and can be fully opened if the duty ratio is greater than or equal to 0.8 . In the paper, the relationship between the engaging velocity of the clutch actuator and the duty ratio is approximately a linear expression. Thus, the engaging velocity of the clutch actuator can be described in equation (20).

$$
v_{e}(k)=v_{e l}+\frac{v_{e h}-v_{e l}}{0.6}\left(c_{e}(k)-0.2\right)
$$


Where $v_{e}(k)$ is the engaging velocity of the clutch actuator at No. $k$ sampling time, $c_{e}(k)$ is the duty ratio of the engaging solenoid at No. $k$ sampling time, $v_{e h}$ is the maximum of the engaging velocity of the clutch actuator which is equal to $20 \mathrm{~mm} / \mathrm{s}$ if the duty ratio is equal to 0.8 , and $v_{e l}$ is the minimum of the engaging velocity of the clutch actuator which is equal to $1 \mathrm{~mm} / \mathrm{s}$ if the duty ratio is equal to 0.2 .

Then, the clutch actuator displacement can be calculated in equation (21) if the engaging solenoid valve is used while the disengaging solenoid valve is closed.

$$
x(k)=x(k-1)+T v_{e}(k-1)
$$

Similarly, the disengaging velocity of the clutch actuator can be described in equation (22).

$$
v_{d}(k)=v_{d l}+\frac{v_{d h}-v_{d l}}{0.6}\left(c_{d}(k)-0.2\right)
$$

Where $v_{d}(k)$ is the disengaging velocity of the clutch actuator at No. $k$ sampling time, $c_{d}(k)$ is the duty ratio of the disengaging solenoid at No. $k$ sampling time, $v_{d h}$ is the maximum of the disengaging velocity of the clutch actuator which is equal to $60 \mathrm{~mm} / \mathrm{s}$ if the duty ratio is equal to 0.8 , and $v_{d l}$ is the minimum of the disengaging velocity of the clutch actuator which is equal to 1 $\mathrm{mm} / \mathrm{s}$ if the duty ratio is equal to 0.2 .

Then, the clutch actuator displacement can be calculated in equation (23) if the disengaging solenoid valve is used while the engaging solenoid valve is closed.

$$
x(k)=x(k-1)-T v_{d}(k-1)
$$

The interval time $T$ is $0.01 \mathrm{~s}$. The belt target velocity under the first gear position is $0.54 \mathrm{~m} / \mathrm{s}$ and the AMT output shaft's target angular speed under the first gear position is $103.18 \mathrm{rpm}$ correspondingly. The maximum value of the belt acceleration is $0.15 \mathrm{~m} / \mathrm{s}^{2}$ and that of the AMT output shaft's equivalent angular acceleration is $3 \mathrm{rad} / \mathrm{s}^{2}$ correspondingly. The ideal time of the acceleration process under the first gear position is $4.8 \mathrm{~s}$ that the ascent acceleration stage or the descent acceleration stage should be $1.2 \mathrm{~s}$ calculated by equation (9) if $\mathrm{N}$ is 4 . The ascent acceleration stage will be finished if the value of the ideal angular acceleration of the AMT output shaft reaches $3 \mathrm{rad} / \mathrm{s}^{2}$. By calculating, the descent acceleration stage will begin if the AMT output shaft's angular speed reaches $85.98 \mathrm{rpm}$. The half engagement point of the clutch actuator for the belt conveyors with full load under the first gear position is $11.35 \mathrm{~mm}$ where the transmitted torque of the clutch is $256.94 \mathrm{~N} \cdot \mathrm{m}$ correspondingly. Considering the delay property of the clutch actuator and avoiding the AMT output shaft's angular acceleration being less than zero during the descent acceleration stage, the duty ratio of the disengaging solenoid valve will be set zero if the clutch actuator displacement is equal to or less than $11.41 \mathrm{~mm}$. To simulate the variation of the belt conveyor's resistance torque in real situations, a white Gaussian noise is added in which the mean and the variance are zero and 10 , respectively.

\section{B. Simulation results based on the prototype MFAC method}

The relevant parameters about the prototype MFAC method during the ascent acceleration stage are described below. The values of $\rho, \eta, \mu$, and $\lambda$ are $1,1,1$, and 0.01 respectively. The initial values of $\phi_{c}(1)$ and $\phi_{c}(2)$ are both 0.5 . The values of $c_{e}(1)$ and $c_{e}(2)$ are both 0.2 . The relevant parameters about the modified MFAC method during the descent acceleration stage are described as follows. The values of $\rho, \eta, \mu$, and $\lambda$ are $1,1,2,0.01$ separately. The values of $\phi_{c}(1)$ and $\phi_{c}(2)$ are both -0.5 . The values of $c_{d}(1)$ and $c_{d}(2)$ are both 0 .

The entire acceleration process is finished in $4.83 \mathrm{~s}$. The times of the ascent, horizontal, and 
descent acceleration stages are finished in $1.20 \mathrm{~s}, 2.16 \mathrm{~s}$, and $1.47 \mathrm{~s}$, respectively. Figures 7, 8, 9, and 10 present the analyses of the control characteristics, the tracking, tracking error, duty ratio, and angular jerk curves, respectively.

As shown in Figure 7, the angular acceleration tracking curve can better track the ideal angular acceleration curve. The absolute maximum of the AMT output shaft's angular acceleration error is $0.22 \mathrm{rad} / \mathrm{s}^{2}$ during the ascent acceleration stage, and that during the descent acceleration stage is $0.26 \mathrm{rad} / \mathrm{s}^{2}$ in Figure 8. The duty ratio of the engaging solenoid valve during the ascent acceleration stage ranges from 0.20 to 0.59 , and that of the disengaging solenoid valve during the descent acceleration stage ranges from 0.20 to 0.51 in Figure 9. The absolute maximum of the AMT output shaft's angular jerk is $30.60 \mathrm{rad} / \mathrm{s}^{3}$ during the ascent acceleration stage, and that during the descent acceleration stage is $32.45 \mathrm{rad} / \mathrm{s}^{3}$ in Figure 10.

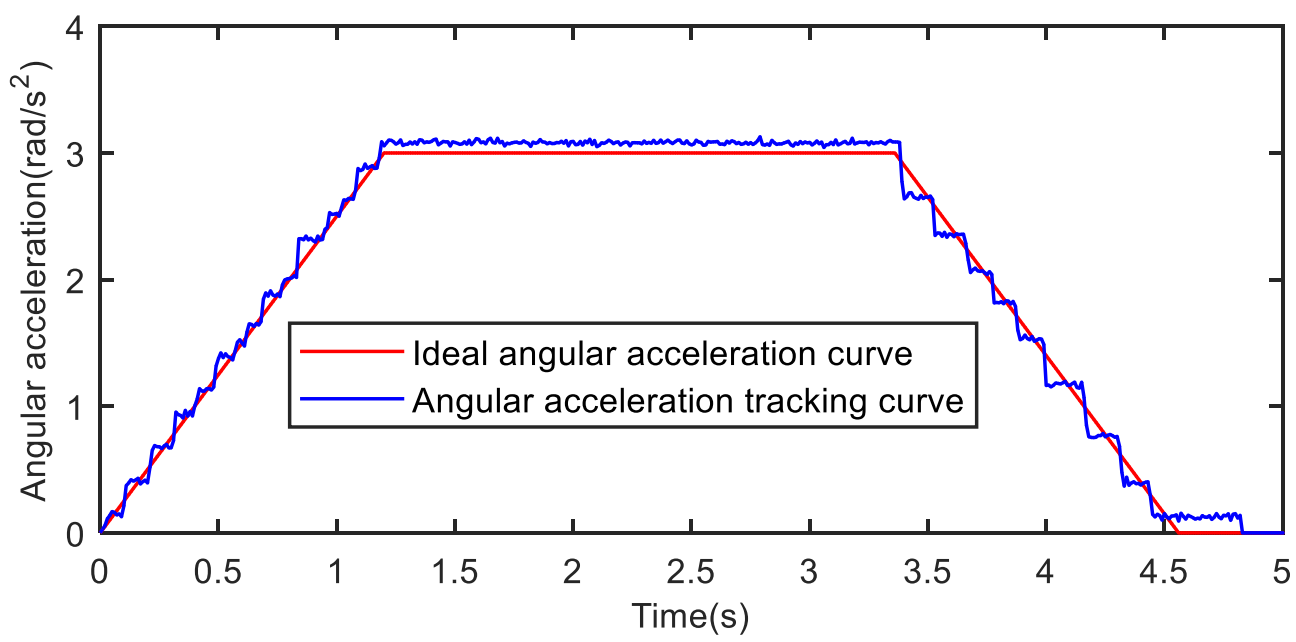

Figure 7. Tracking curve based on the prototype MFAC method.

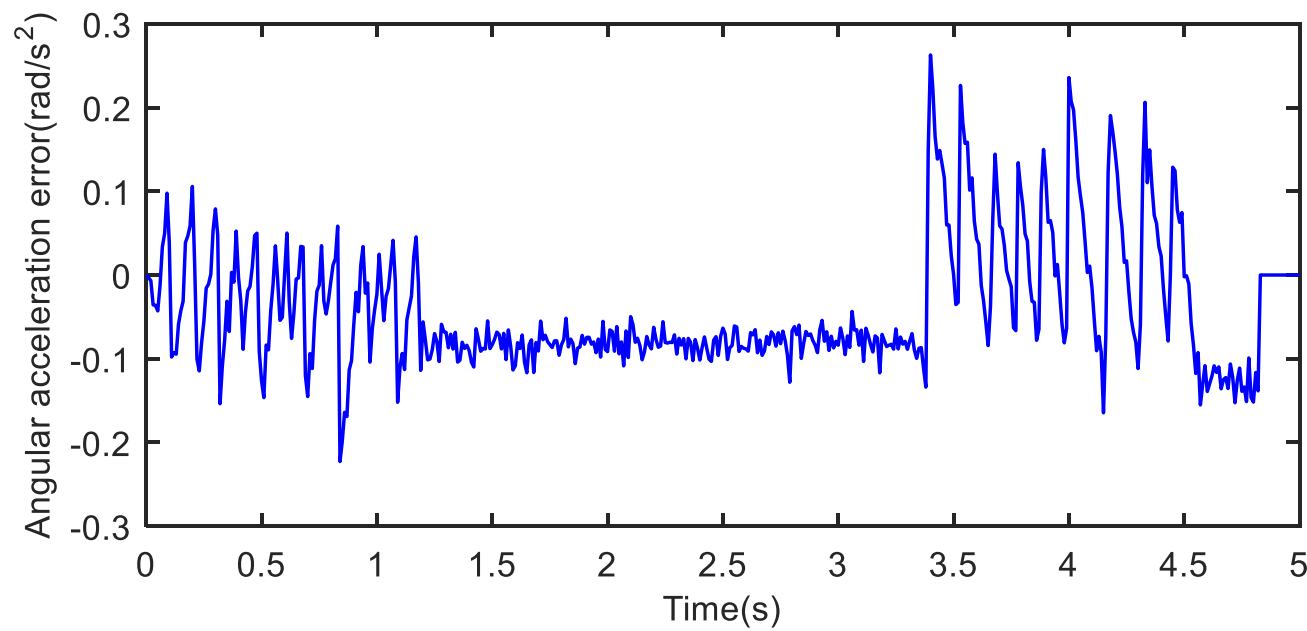

Figure 8. Tracking error curve based on the prototype MFAC method. 


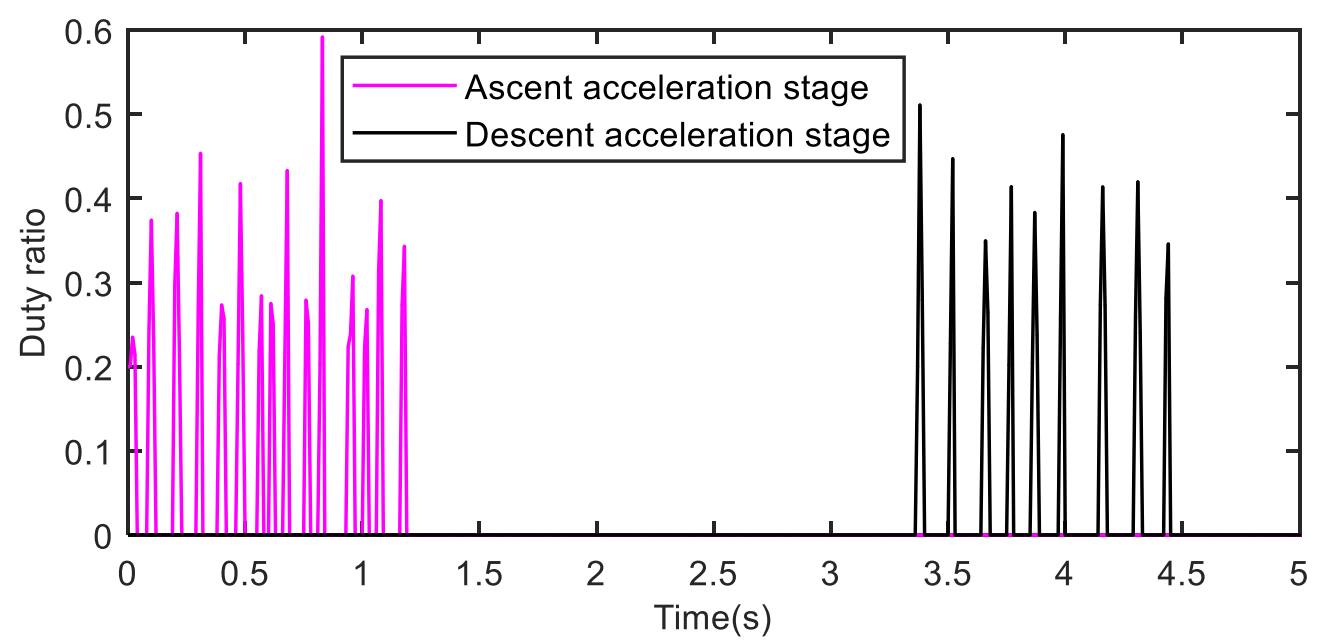

Figure 9. Duty ratio curve based on the prototype MFAC method.

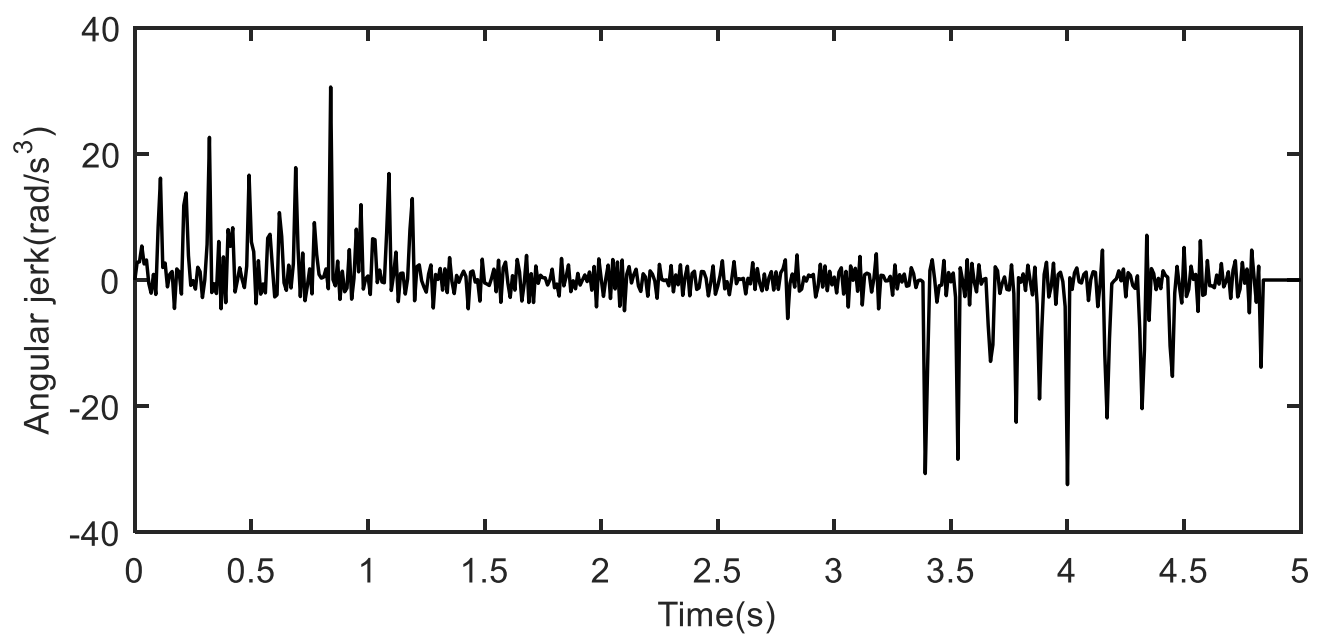

Figure 10. Angular jerk curve based on the prototype MFAC method.

\section{Simulation results based on the modified MFAC method with jerk}

\section{compensation}

The related parameters about the modified MFAC method with jerk compensation are identical to those about the prototype MFAC method. The values of $\ddot{\omega}_{\text {omax }}$ and $\xi$ are $20 \mathrm{rad} / \mathrm{s}^{3}$ and 0.5 , respectively. The entire acceleration process is finished in 4.90s. The times of the ascent, horizontal and descent acceleration stages are finished in $1.20 \mathrm{~s}, 2.22 \mathrm{~s}$, and $1.48 \mathrm{~s}$ respectively. To show the advantages of the modified MFAC method with jerk compensation, Figures 11, 12, 13, and 14 show the tracking, tracking error, duty ratio curve, and angular jerk curves, respectively.

Figure 11 shows that the angular acceleration tracking curve can track the modified ideal angular acceleration curve well. The absolute maximum of the AMT output shaft's angular acceleration error is $0.17 \mathrm{rad} / \mathrm{s}^{2}$ during the ascent acceleration stage, and that during the descent acceleration stage is $0.18 \mathrm{rad} / \mathrm{s}^{2}$ in Figure 12. The range of duty ratio of the engaging solenoid valve during the ascent acceleration stage is from 0.2 to 0.46 , and that of the disengaging solenoid valve during the descent acceleration stage is from 0.2 to 0.40 in Figure 13. The AMT output shaft's angular jerk curve can be efficiently controlled in Figure 14, in which the absolute maximum of the AMT output shaft's angular jerk is $21.87 \mathrm{rad} / \mathrm{s}^{3}$ during the ascent acceleration stage, and that during the descent 
acceleration stage is $21.79 \mathrm{rad} / \mathrm{s}^{3}$. Obviously, the absolute maximum of the AMT output shaft's angular jerk based on the modified MFAC method with jerk compensation in Figure 14 is less than that based on the prototype MFAC in Figure 10. Specifically, the modified MFAC method with jerk compensation can control the AMT output shaft's angular acceleration and the AMT output shaft's angular jerk better.

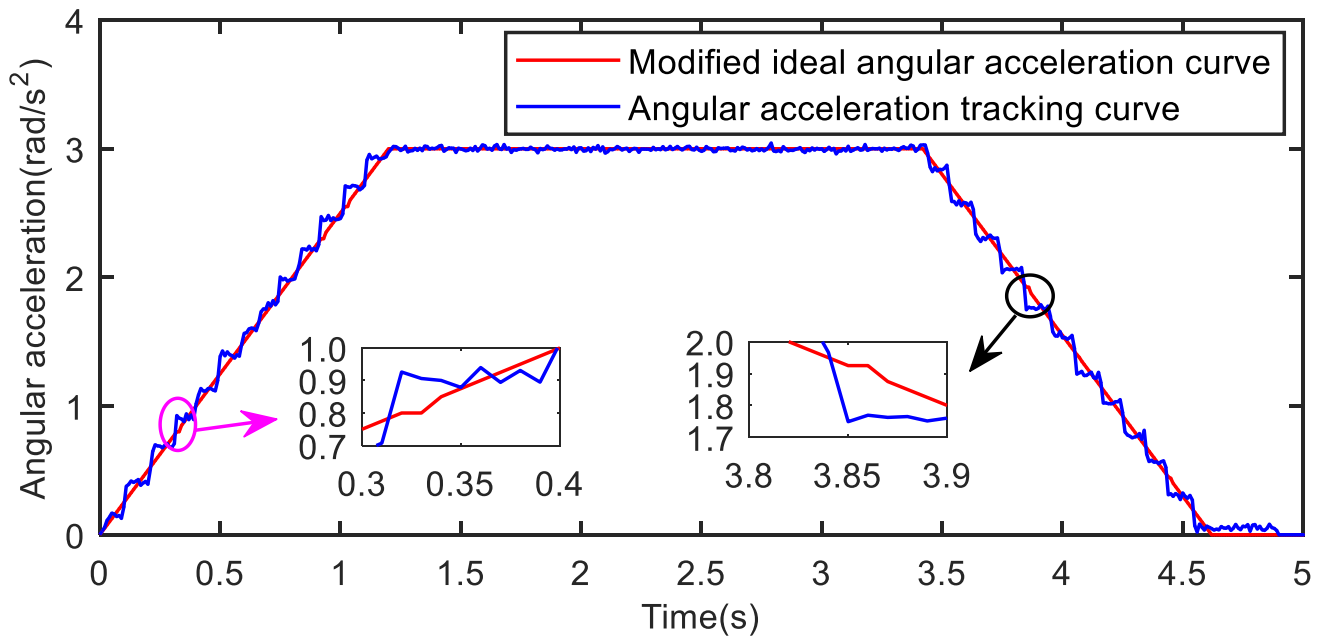

Figure 11. Tracking curve based on the modified MFAC method with jerk compensation.

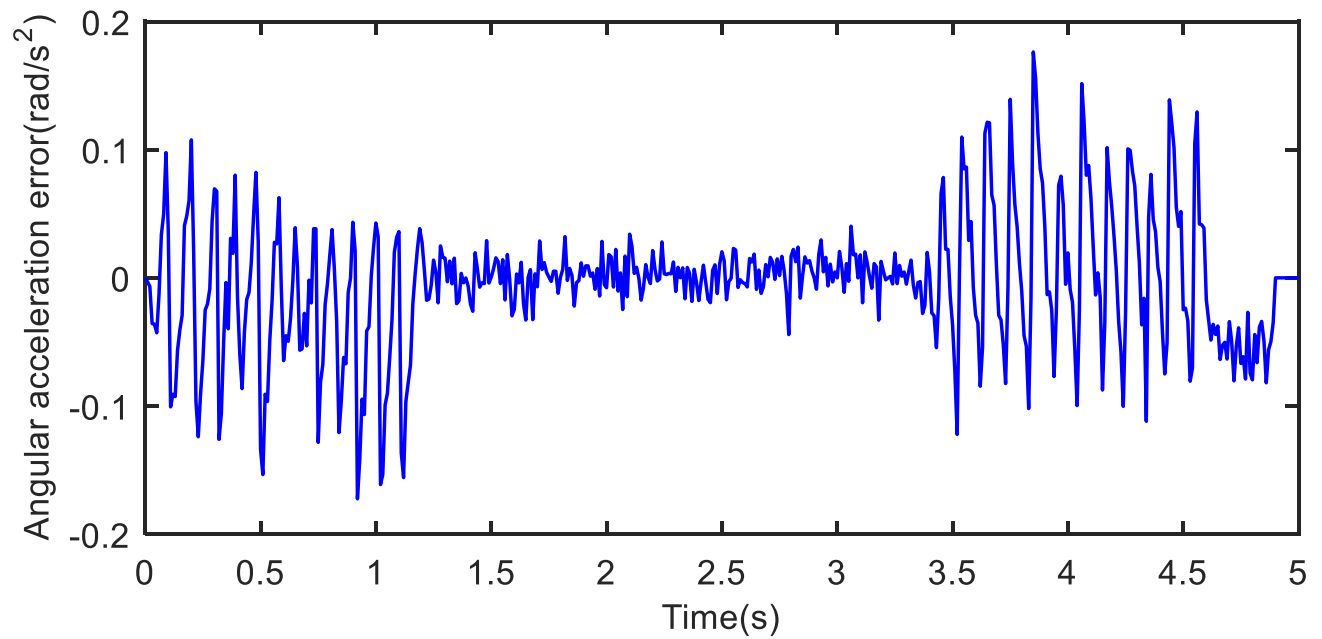

Figure 12. Tracking error curve based on the modified MFAC method with jerk compensation.

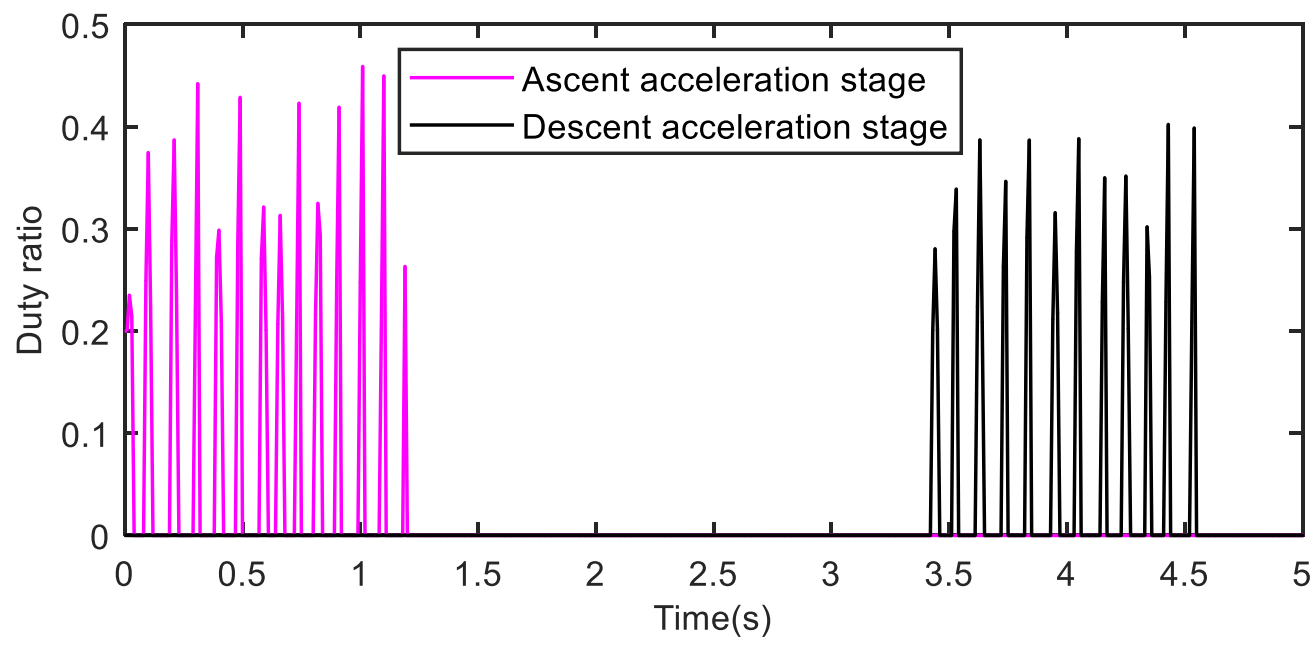

Figure 13. Duty ratio curve based on the modified MFAC method with jerk compensation. 


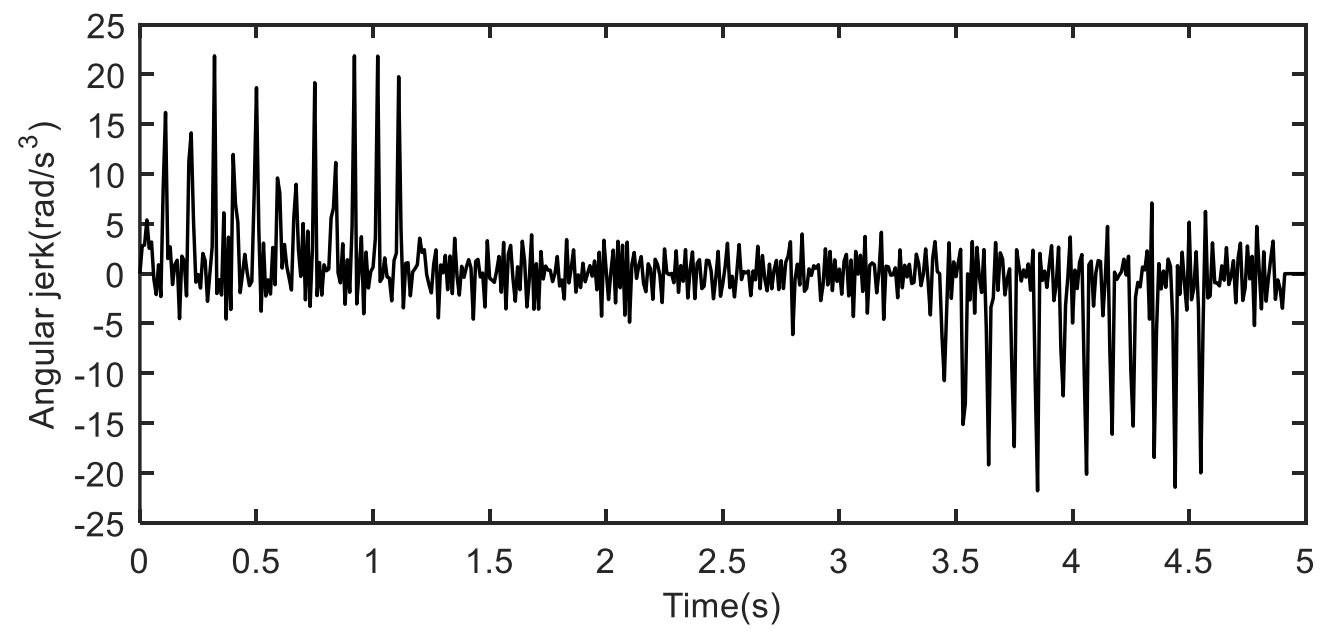

Figure 14. Angular jerk curve based on the modified MFAC method with jerk compensation.

\section{Simulation results based on the PID control method}

To illustrate the advantages of the MFAC method, the simulation results based on the PID method are given under the same condition of full load. After repeated tests, the better PID parameters are obtained: the proportion coefficient is 5 , the integral coefficient is zero, and the differential coefficient is 0.01 during both the ascent and descent acceleration stages. The acceleration process is finished in 4.82s. The ascent, horizontal, and descent acceleration stages are finished in $1.20 \mathrm{~s}$, $2.27 \mathrm{~s}$, and $1.35 \mathrm{~s}$, respectively. Following the PID control method, Figures 15, 16, 17, and 18 present the tracking, tracking error, duty ratio, and angular jerk curves, respectively.

As shown in Figure 15, the angular acceleration tracking curve can sensitively track the ideal angular acceleration curve. The absolute maximum of the AMT output shaft's angular acceleration tracking error is $0.22 \mathrm{rad} / \mathrm{s}^{2}$ during the ascent acceleration stage, while that during the descent acceleration stage is $0.32 \mathrm{rad} / \mathrm{s}^{2}$ in Figure 16, which is obviously greater than that based on the prototype MFAC method in Figure 8 and that based on the modified MFAC method with jerk compensation in Figure 12. The duty ratio of the engaging solenoid valve during the ascent acceleration stage changes from 0.2 to 0.55 , and that during the descent acceleration stage changes from 0.2 to 0.59 in Figure 17, which is obviously greater than that based on the prototype MFAC method and that based on the modified MFAC method. The absolute maximum of the AMT output shaft's angular jerk based on the PID method during the ascent acceleration stage is $28.11 \mathrm{rad} / \mathrm{s}^{3}$, and that during the descent acceleration stage is $44.40 \mathrm{rad} / \mathrm{s}^{3}$ in Figure 18. By contrast, the absolute maximum of the AMT output shaft's angular jerk based on the PID method is obviously greater in Figure 18 than that based on the prototype MFAC method in Figure 10 and that based on the modified MFAC method with jerk compensation in Figure 14. 


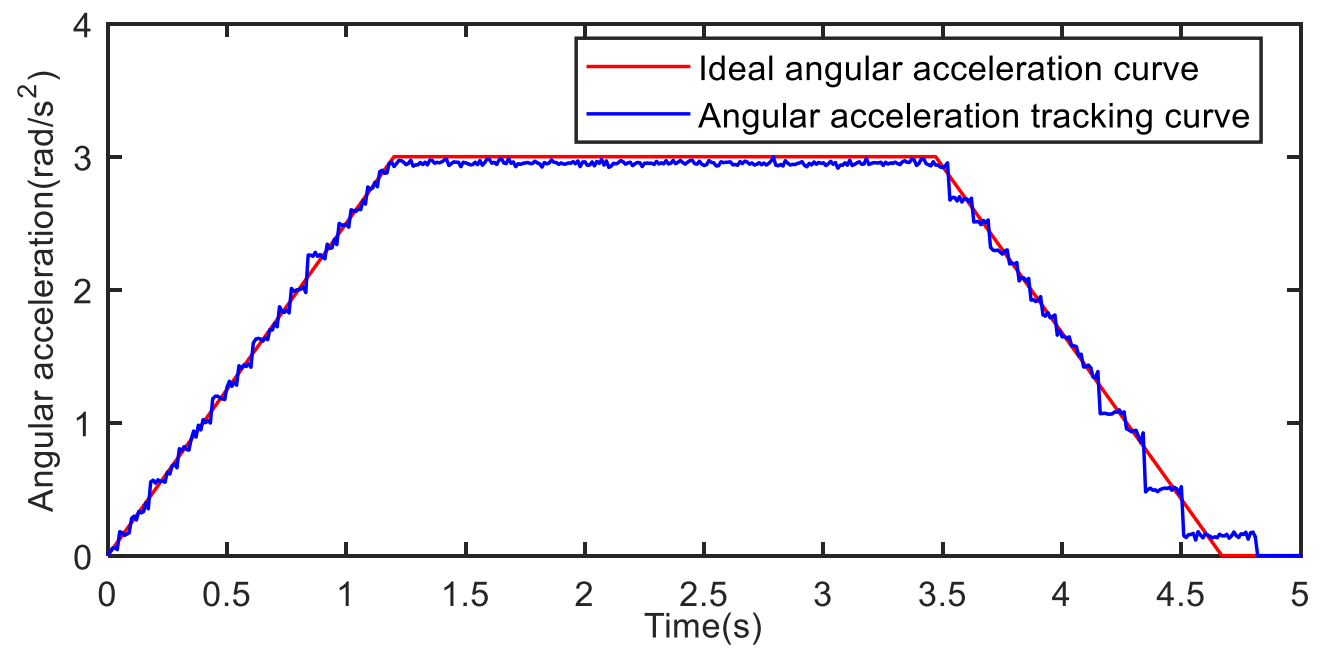

Figure 15. Tracking curve based on the PID method.

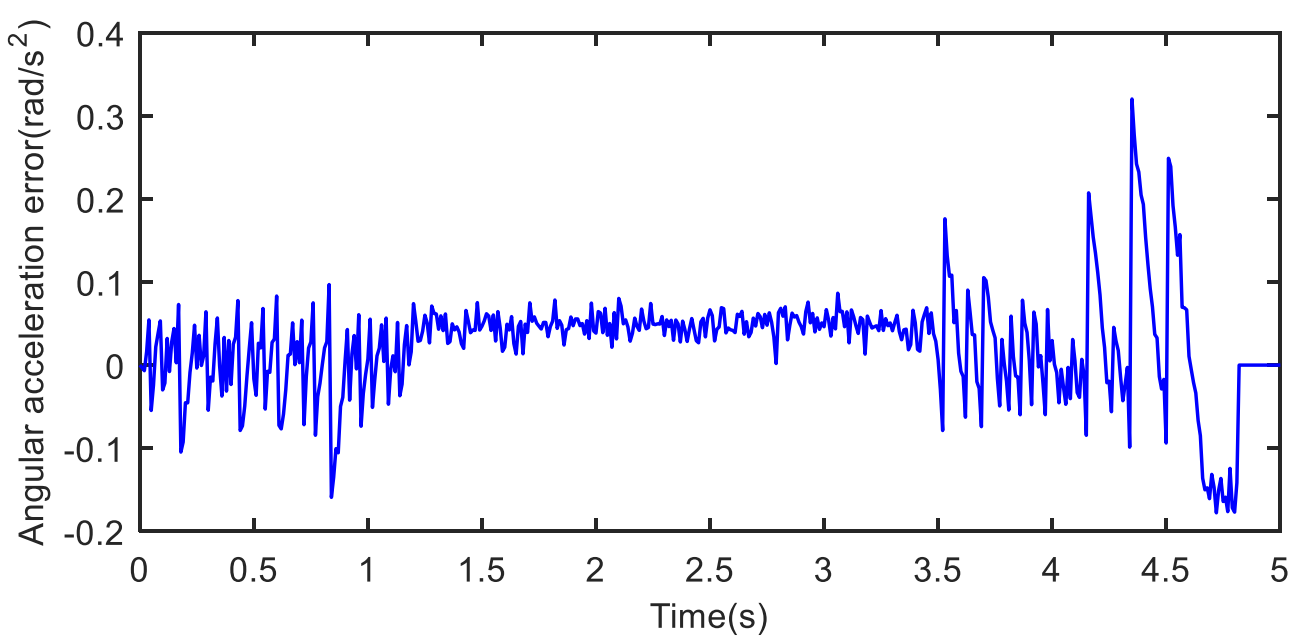

Figure 16. Tracking error curve based on the PID method.

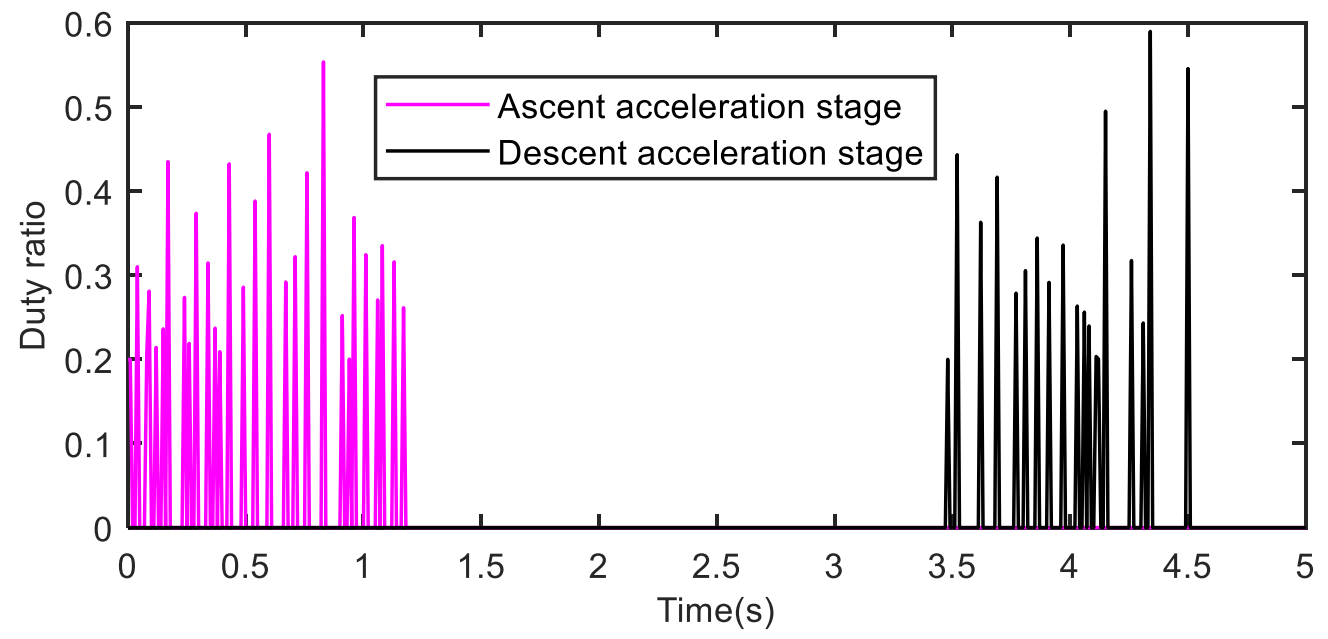

Figure 17. Duty ratio curve based on the PID method. 


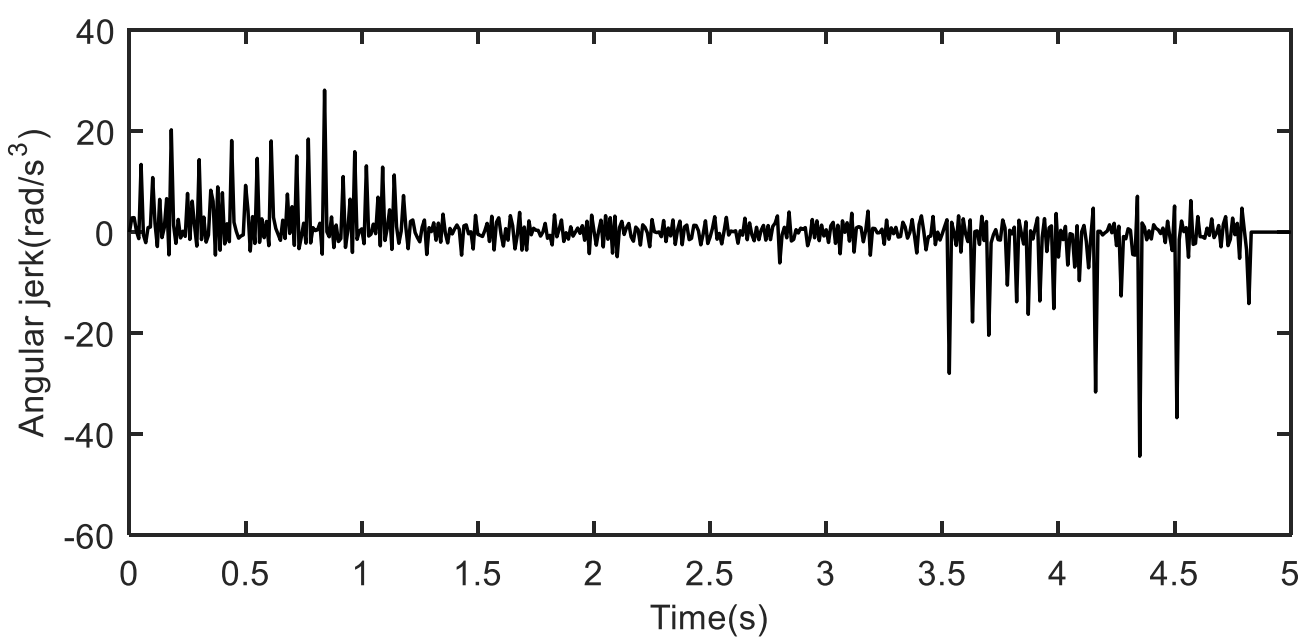

Figure 18. Angular jerk curve based on the PID method.

\section{E. Comparisons of the three control methods}

Table 2 lists the main data comparisons among the three data-driven methods.

Table 2. Data comparisons among the three data-driven methods

\begin{tabular}{|c|c|c|c|c|}
\hline Characteristic parameter & Stage & $\begin{array}{l}\text { Modified MFAC } \\
\text { method with jerk } \\
\text { compensation }\end{array}$ & $\begin{array}{c}\text { Prototype } \\
\text { MFAC }\end{array}$ & PID \\
\hline Absolute maximum of the & & & & \\
\hline $\begin{array}{l}\text { AMT output shaft's } \\
\text { angular acceleration error }\end{array}$ & Ascent stage & 0.17 & 0.22 & 0.22 \\
\hline $\begin{array}{l}\text { Absolute maximum of the } \\
\text { AMT output shaft's } \\
\text { angular acceleration error }\end{array}$ & Descent stage & 0.17 & 0.26 & 0.32 \\
\hline $\begin{array}{l}\text { Absolute maximum of the } \\
\text { AMT output shaft's } \\
\text { angular jerk }\left(\mathrm{rad} / \mathrm{s}^{3}\right)\end{array}$ & Ascent stage & 21.87 & 30.60 & 28.11 \\
\hline $\begin{array}{l}\text { Absolute maximum of the } \\
\text { AMT output shaft's } \\
\text { angular jerk }\left(\mathrm{rad} / \mathrm{s}^{3}\right)\end{array}$ & Descent stage & 21.79 & 32.45 & 44.40 \\
\hline
\end{tabular}

As seen from Table 2, the absolute maximum of the AMT output shaft's angular acceleration tracking error based on the modified MFAC method with jerk compensation is lower than the other two control methods. By calculating, the absolute maximum of the AMT output shaft's angular acceleration tracking error based on the modified MFAC method with jerk compensation accounts for approximately $77.41 \%$ of that based on the prototype MFAC method and the PID control method during the ascent acceleration stage. In addition, the absolute maximum of the angular acceleration tracking error accounts for approximately $67.09 \%$ of that based on the prototype MFAC method and approximately $55.04 \%$ of that based on the PID control method during the descent acceleration stage. Thus, the modified MFAC method with jerk compensation is an optimal control method to track the ideal angular acceleration curve.

Moreover, Table 2 indicates that the absolute maximum of the AMT output shaft's angular jerk 
based on the modified MFAC method with jerk compensation is lower than that based on the prototype MFAC method and that the PID control method. By calculating, the absolute maximum of the AMT output shaft's angular jerk based on the modified MFAC method with jerk compensation accounts for approximately $71.16 \%$ of that based on the prototype MFAC method and approximately $77.79 \%$ of that based on the PID method during the ascent acceleration stage. Furthermore, the absolute maximum of the AMT output shaft's angular jerk based on the modified MFAC method with jerk compensation accounts for approximately $67.16 \%$ of that based on the prototype MFAC method and approximately $49.08 \%$ of that based on the PID method during the descent acceleration stage. Consequently, the absolute maximum of the AMT output shaft's angular jerk based on the modified MFAC method with jerk compensation is the least, that of the AMT output shaft's angular jerk based on the prototype MFAC method is bigger, and that of the AMT output shaft's angular jerk based on the PID control method is the largest. The jerk compensation is proven effective for reducing the transmission shock.

In sum, the modified MFAC method with jerk compensation can clearly enhance the reduction of the AMT output shaft's angular jerk and has smaller angular acceleration tracking error. Thus, the modified MFAC method with jerk compensation can be used as the appropriate method for controlling the AMT output shaft's angular acceleration and angular jerk, providing better performances for AMT as a soft starter.

\section{Conclusions}

A new data-driven control method named the modified MFAC method with jerk compensation is studied for AMT as the soft starter in the paper. First, based on the clutch characteristics, using the segmented acceleration curve comprising several trapezoidal acceleration curves is proposed as the ideal acceleration curve for AMT as the soft starter. Second, the dynamic analysis of AMT is discussed. Third, based on the prototype MFAC method, the modified MFAC method with jerk compensation for controlling the AMT output shaft's angular acceleration is proposed. Finally, the simulation results are analyzed on the bases of the three data-driven methods including the prototype MFAC method, the modified MFAC method with jerk compensation, and the PID control method. The results confirm its effectiveness for using the modified MFAC method with jerk compensation to control the AMT output shaft's angular acceleration with less tracking error and smaller shock.

\section{Acknowlegements}

This work was supported by Key R \& D project of Shandong Province, China (Grant No. 2020CXGC011003).

\section{References}

[1] W. Wang, Y. Deng, L. Guo, The dynamic design method of the belt conveyor basing on the power track control strategy, Dongbei Daxue Xuebao/Journal of Northeastern University, vol. 23, no. 9, pp. 899-902, 2002.

[2] L. K. Nordell, Z. P. Ciozda, Transient belt stresses during starting and stopping: elastic response simulated by finite element methods, Bulk Solids Handling, vol. 4, no. 1, pp. 93-98, 1984.

[3] P. Xi, H. Zhang, J. Liu, Dynamics simulation of the belt conveyor possessing feedback loop during starting, Journal of Coal Science and Engineering, vol. 11, no. 1, pp. 83-85, 2005.

[4] G. Wang, L. Zhang, H. Sun, C. Zhu, Longitudinal tear detection of conveyor belt under uneven 
light based on Haar-AdaBoost and Cascade algorithm, MEASUREMENT, vol. 168, Article ID. 108341, 2021.

[5] R. Yang T. Qiao, Y. Pang, Infrared spectrum analysis method for detection and early warning of longitudinal tear of mine, MEASUREMENT, vol. 165, Article ID. 107856, 2020.

[6] J. Ji, C. Miao, X. Li, Cosine-trapezoidal soft-starting control strategy for a belt conveyor, Mathematical Problems in Engineering, vol. 2019, Article ID. 8164247, 2019.

[7] M. H. Ghayesh, Nonlinear transversal vibration and stability of an axially moving viscoelastic string supported by a partial viscoelastic guide, Journal of Sound and Vibration, vol. 314, no. 3-5, pp. 757-774, 2008.

[8] D. S. Mallick, A tricky problem: selection of drive systems for long-distance conveyor systems, Bulk Solids Handling, vol. 33, no. 5, pp. 28-31, 2013.

[9] Q. Meng, Y. Hou, Effect of oil film squeezing on hydro-viscous drive speed regulating start, Tribology International, vol. 43, no. 11, pp. 2134-2138, 2010.

[10] M. Zhou, C. Wei, Y. Yu, Y. Zhang, Oil-film clutch in soft-start of belt conveyor, Journal of Beijing Institute of Technology (English Edition), vol. 11, no. 2, pp. 142-145 2002.

[11] H. Wang, B. Wang, D. Pi, Two-layer structure control of an automatic mechanical transmission clutch during hill start for heavy-duty vehicles, IEEE ACCESS, vol. 8, pp. 49617-49628, 2020.

[12] B. Gao, X. Lu, H. Chen, Dynamics and control of gear upshift in automated manual transmissions, International Journal of Vehicle Design, vol. 63, no. 1, pp. 61-83, 2013.

[13] Y. Li, Z. Wang, X. Cong, Constant acceleration control for AMT's application in belt conveyor's soft starting, International Journal of Control and Automation, vol. 6, no. 6, pp. 315326, 2013.

[14] Y. Li, Y. Lu, H. Sun, P. Yan, Study on load parameters estimation during AMT startup, Journal of Control Science and engineering, vol. 2018, Article ID 7391968, 2018.

[15] Z. Zhao, J. Gu, L. He, Estimation of torques transited by twin-clutch during shifting process for dry dual clutch transmission, Journal of Mechanical Engineering, vol. 53, no. 14, pp. 77-87, 2017.

[16] Z. Zhao, L. He, Y. Yang, C. Wu, X. Li, J. K. Hedrick, Estimation of torque transmitted by clutch during shifting process for dry dual clutch transmission, Mechanical Systems and Signal Processing, vol. 75, pp. 413-433, 2016.

[17] Z. Zhao, C. Wu, Y. Yang, H. Chen, Optimal robust control of shifting process for hybrid electric car with dry dual clutch transmission, Journal of Mechanical Engineering, vol. 52, no. 18, pp. 105117, 2016.

[18] H. He, T. Si, L. Sun, B. Liu, Z. Li, Linear active disturbance rejection control for three-phase voltage-source PWM rectifier, IEEE Access, vol. 8, pp. 45050-45060, 2020.

[19] P. V. Pakshin, A. S. Koposov, J. P. Emelianova, Iterative learning control of a multiagent system under random perturbations, Automation and Remote Control, vol. 81, no. 3, pp. 483-502, 2020.

[20] S. Liu, Z. Hou, T. Tian, Z. Deng, Z. Li, A novel dual successive projection-based model-free adaptive control method and application to an autonomous car, IEEE Transactions on Neural Networks and Learning Systems, vol. 30, no. 11, pp. 3444-3457, 2019.

[21] Z. Hou, S. Xiong, On model-free adaptive control and its stability analysis, IEEE Transactions on Automatic Control, vol. 64, no. 11, pp. 4555-4569, 2019.

[22] Z. Yan, F. Yan, J. Liang, Y. Duan, Detailed Modeling and experimental assessments of automotive dry clutch engagement, IEE Access, vol. 7, pp. 59100-59113, 2019. 
[23] Q. Chen, D. Qin, X. Ye, Optimal control about AMT heavy-duty truck starting clutch, Zhongguo Gonglu Xuebao/China Journal of Highway and Transport, vol. 23, no. 1, pp. 116-121, 2010. 
Figures

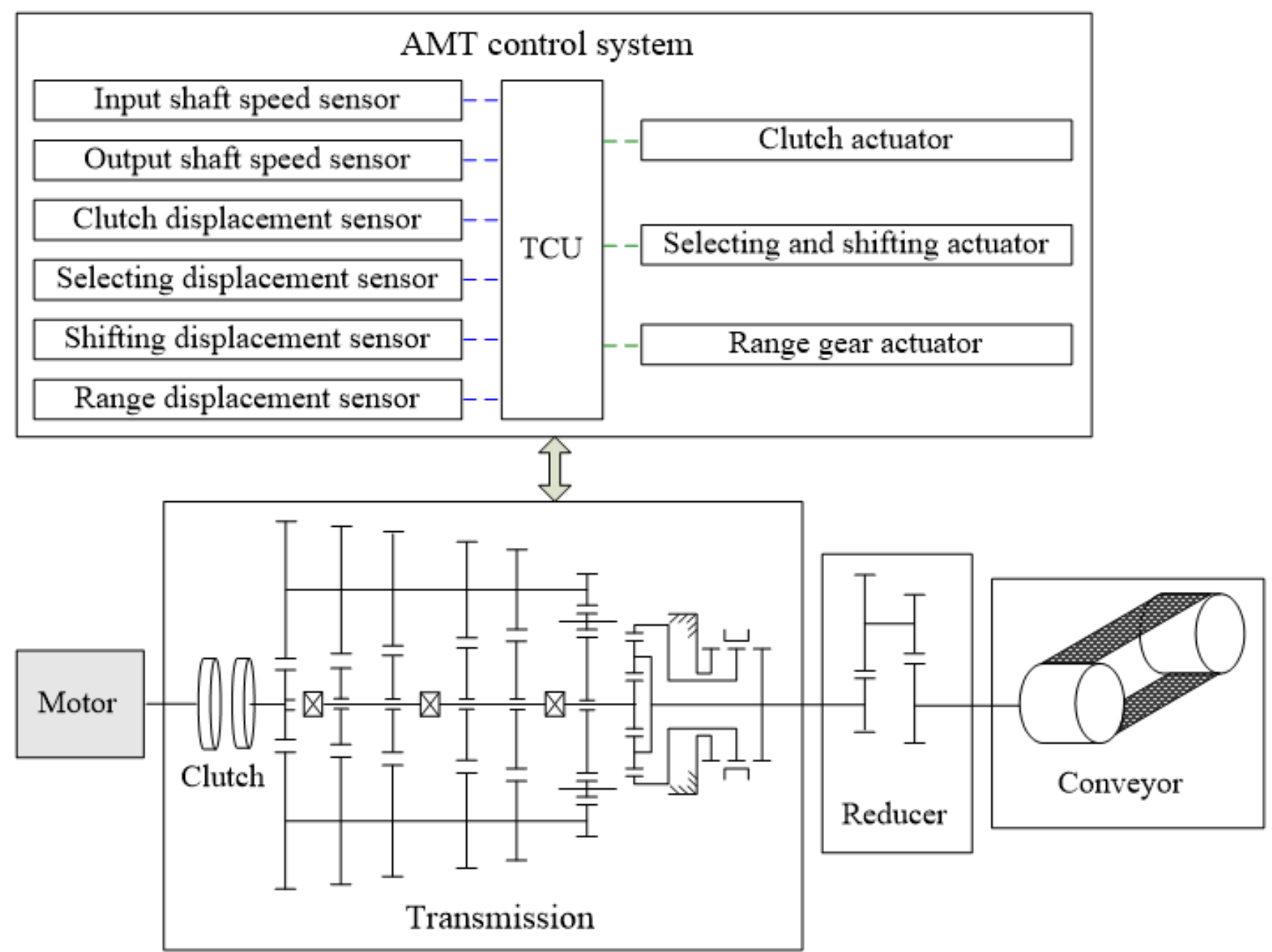

Figure 1

Diagram of AMT soft-starting system. 


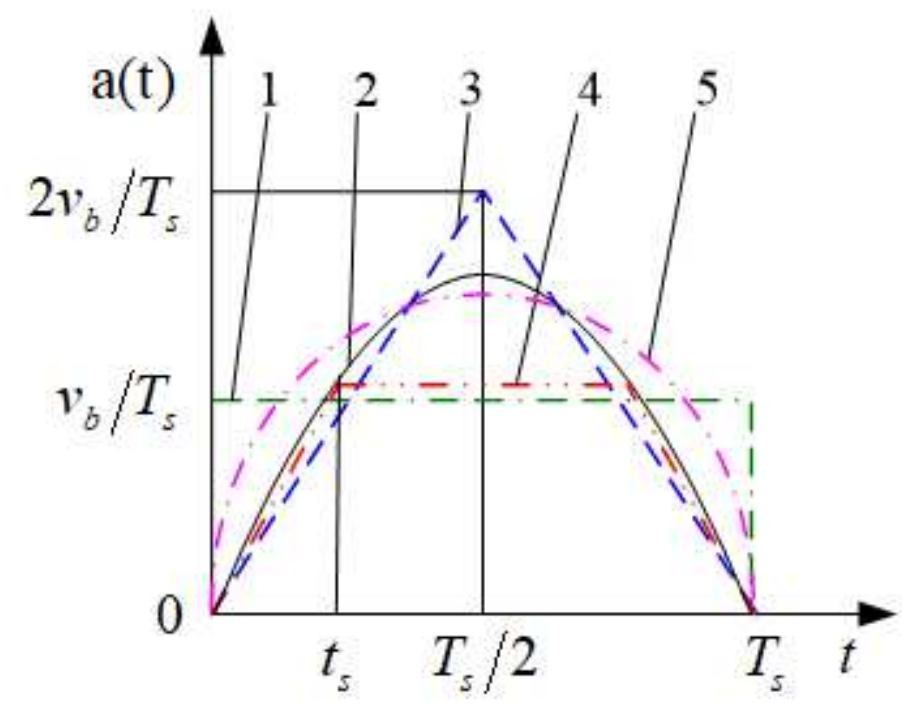

Figure 2

Five common starting acceleration curves.

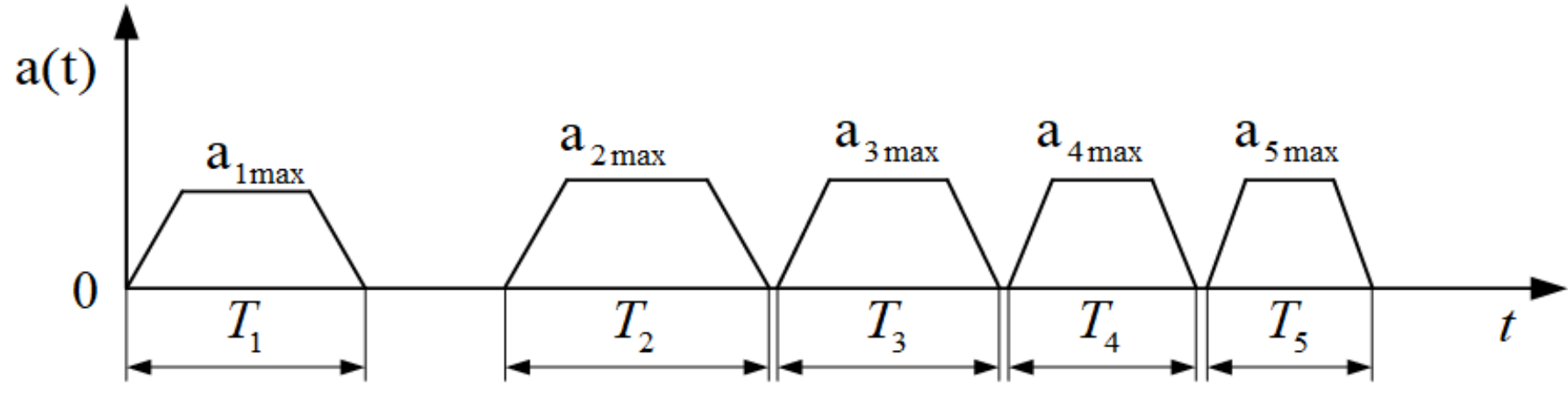

Figure 3

Starting acceleration curve based on AMT. 


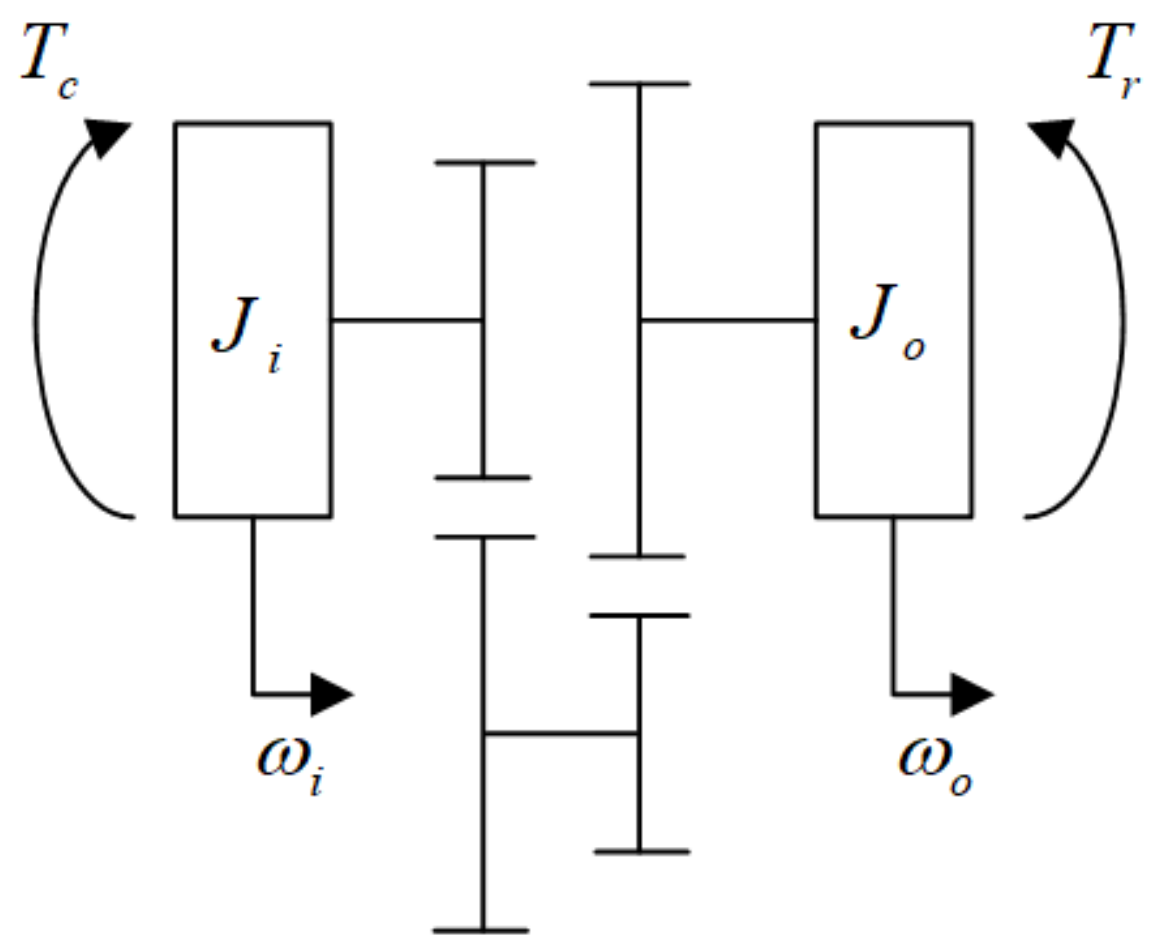

Figure 4

Transmission double-mass model.

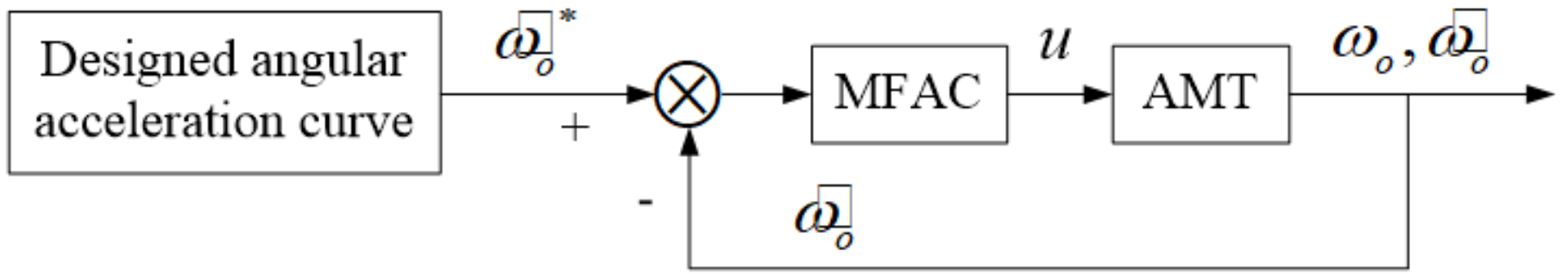

Figure 5

Block diagram of the prototype MFAC method.

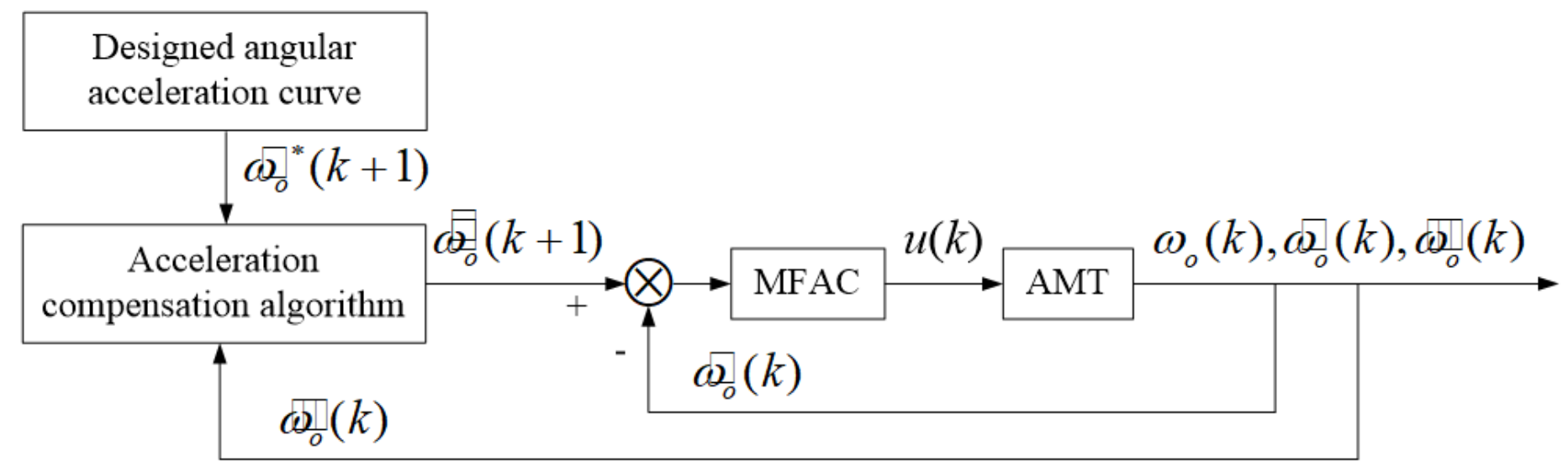


Figure 6

Block diagram of the MFAC method with jerk compensation.

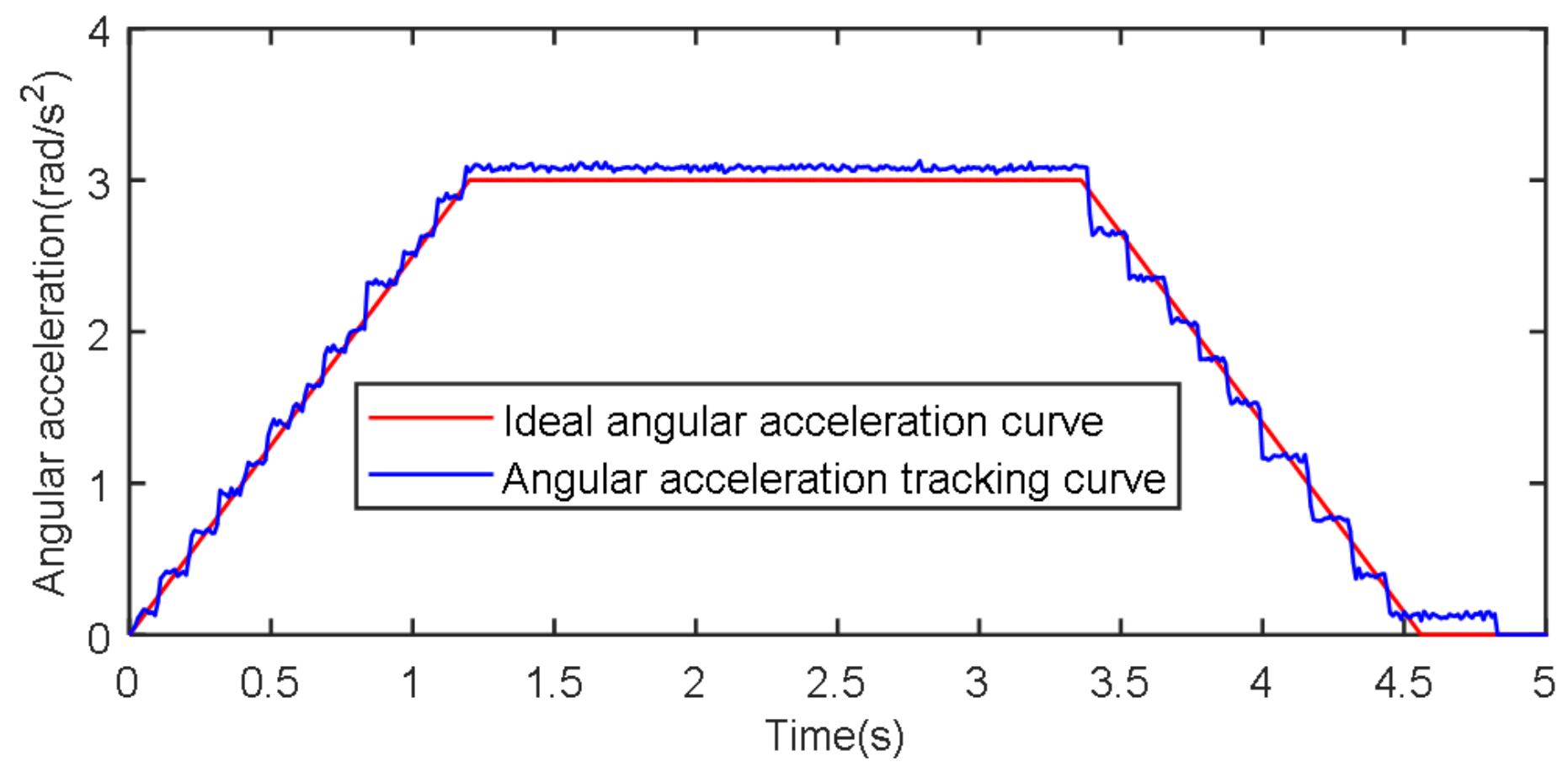

Figure 7

Tracking curve based on the prototype MFAC method.

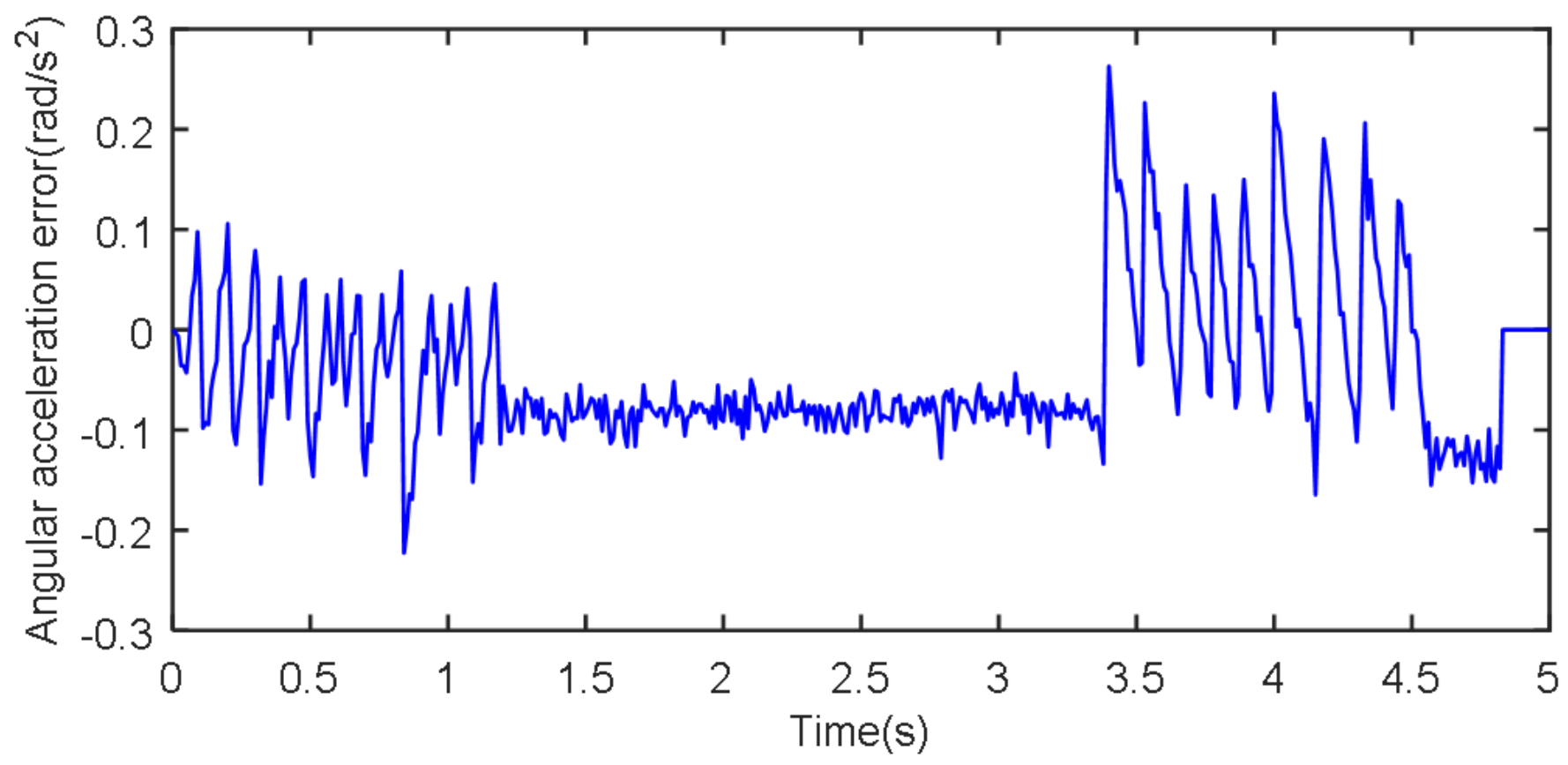

Figure 8

Tracking error curve based on the prototype MFAC method. 


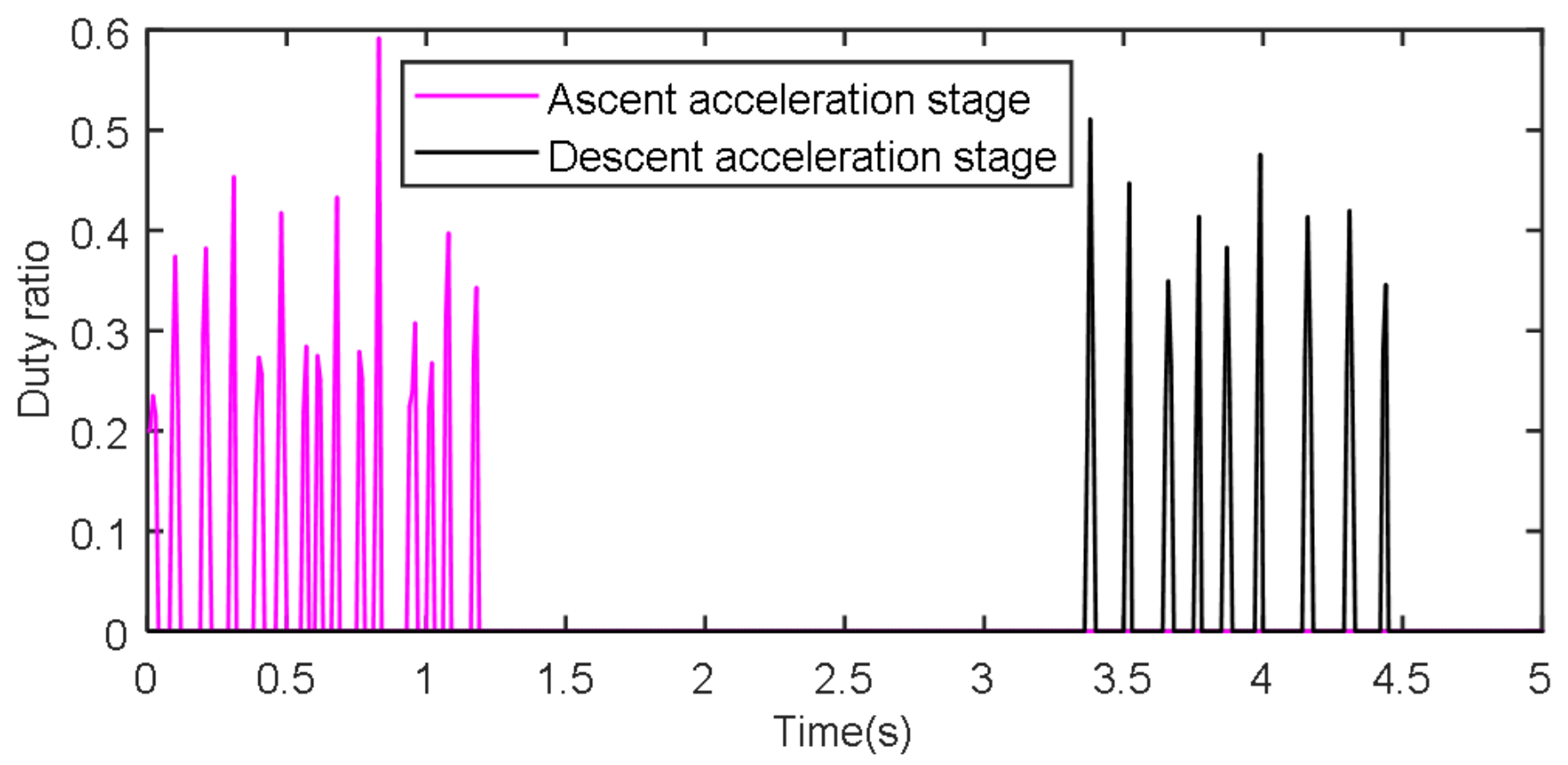

Figure 9

Duty ratio curve based on the prototype MFAC method.

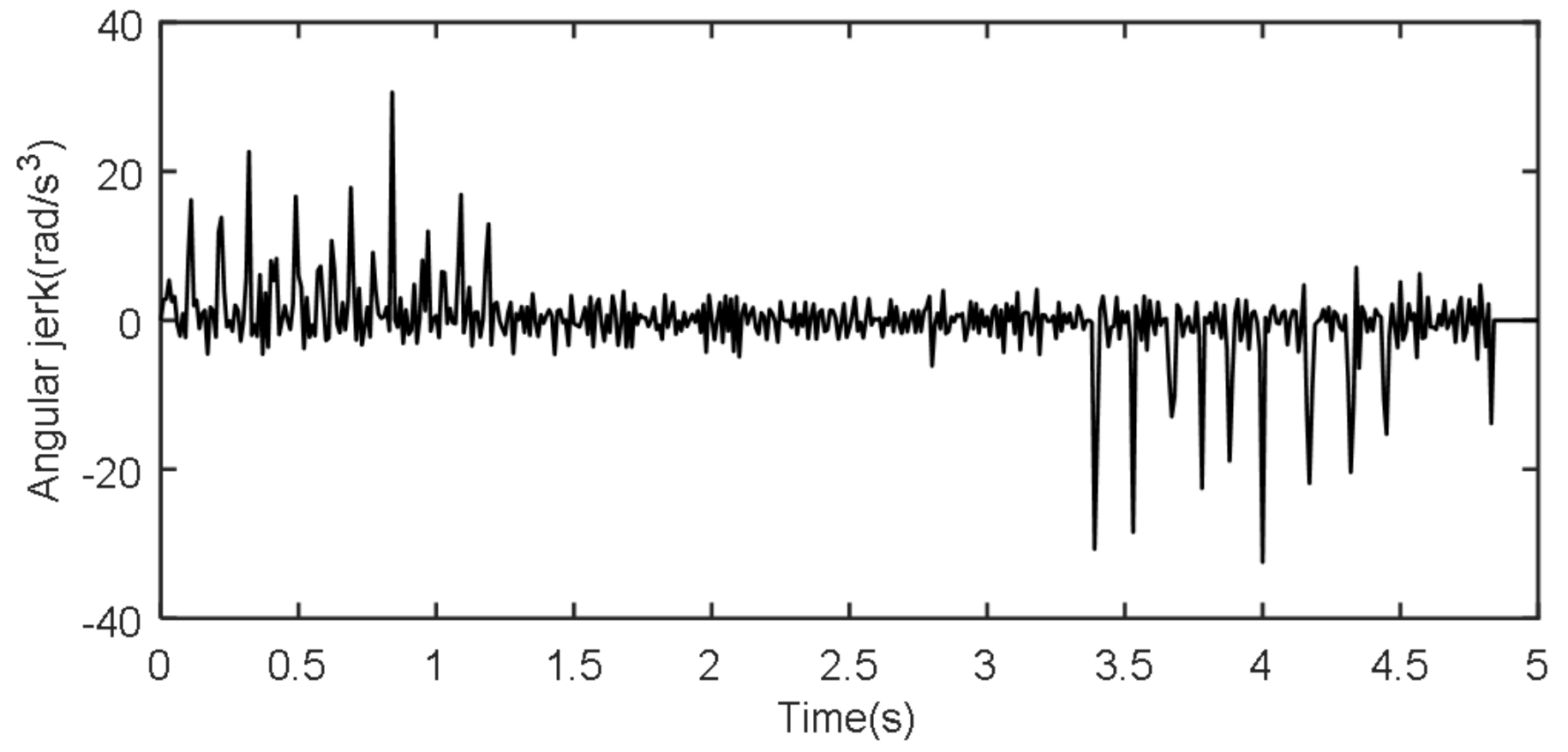

Figure 10

Angular jerk curve based on the prototype MFAC method. 


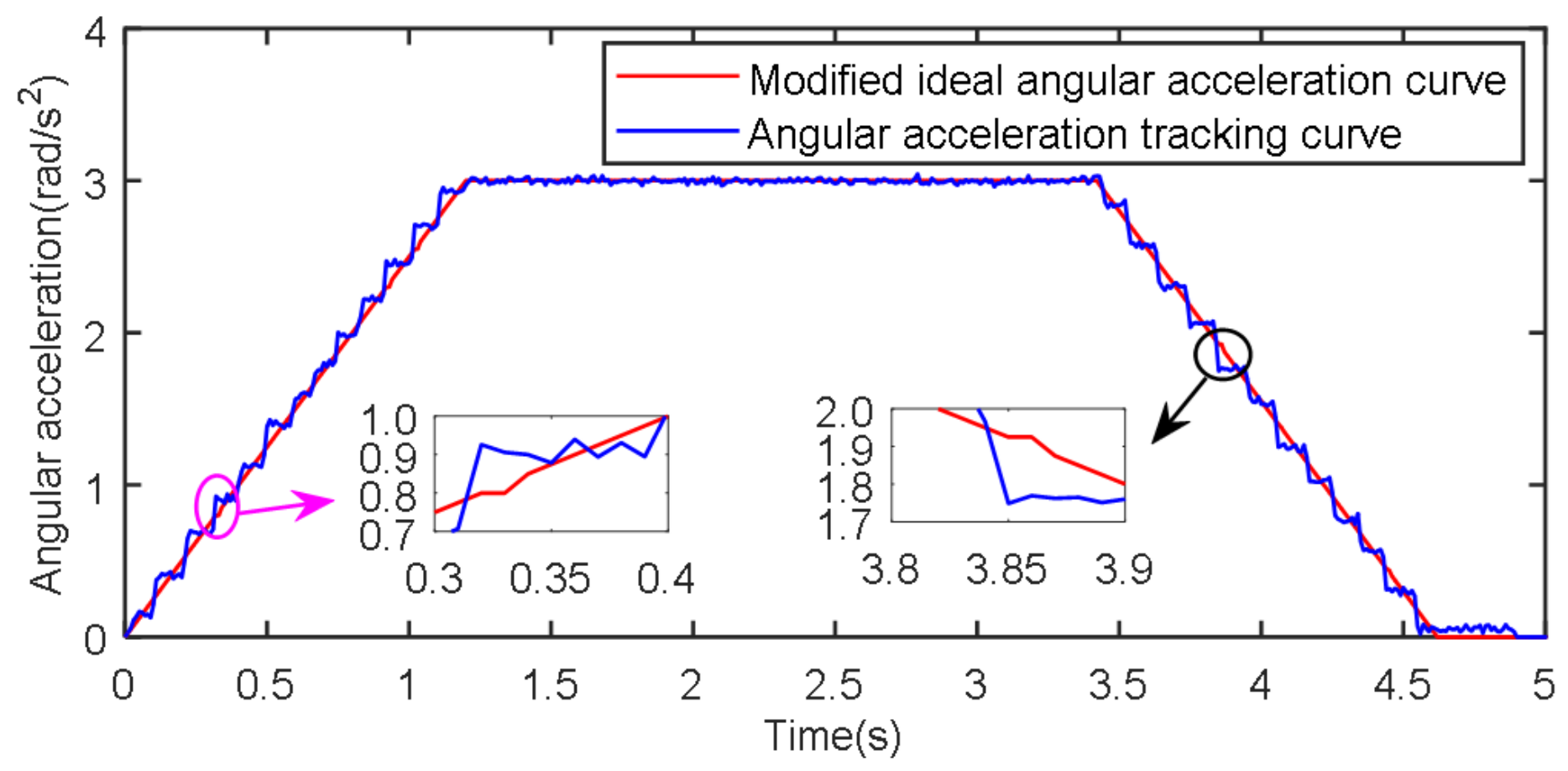

Figure 11

Tracking curve based on the modified MFAC method with jerk compensation.

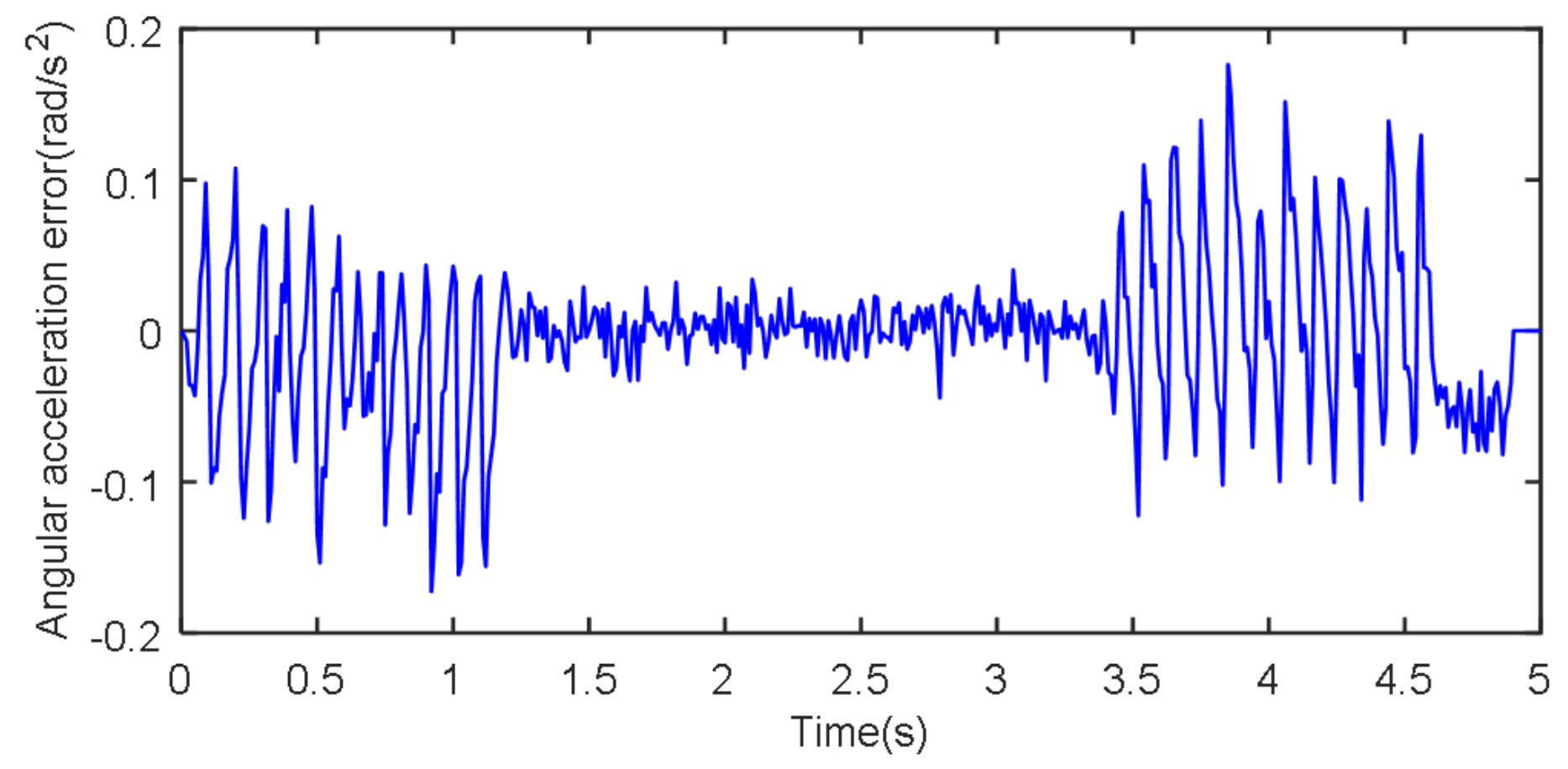

Figure 12

Tracking error curve based on the modified MFAC method with jerk compensation. 


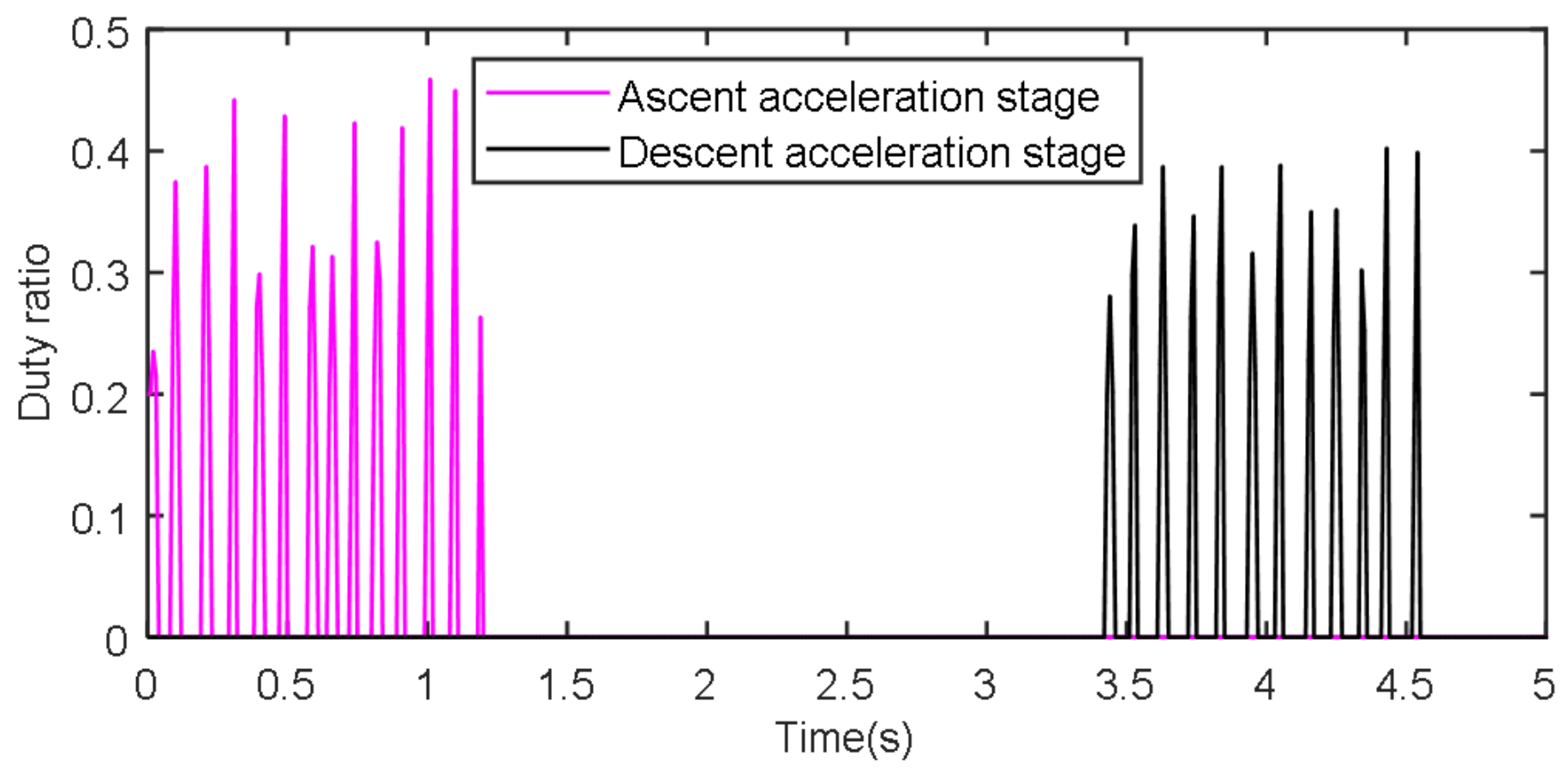

Figure 13

Duty ratio curve based on the modified MFAC method with jerk compensation.

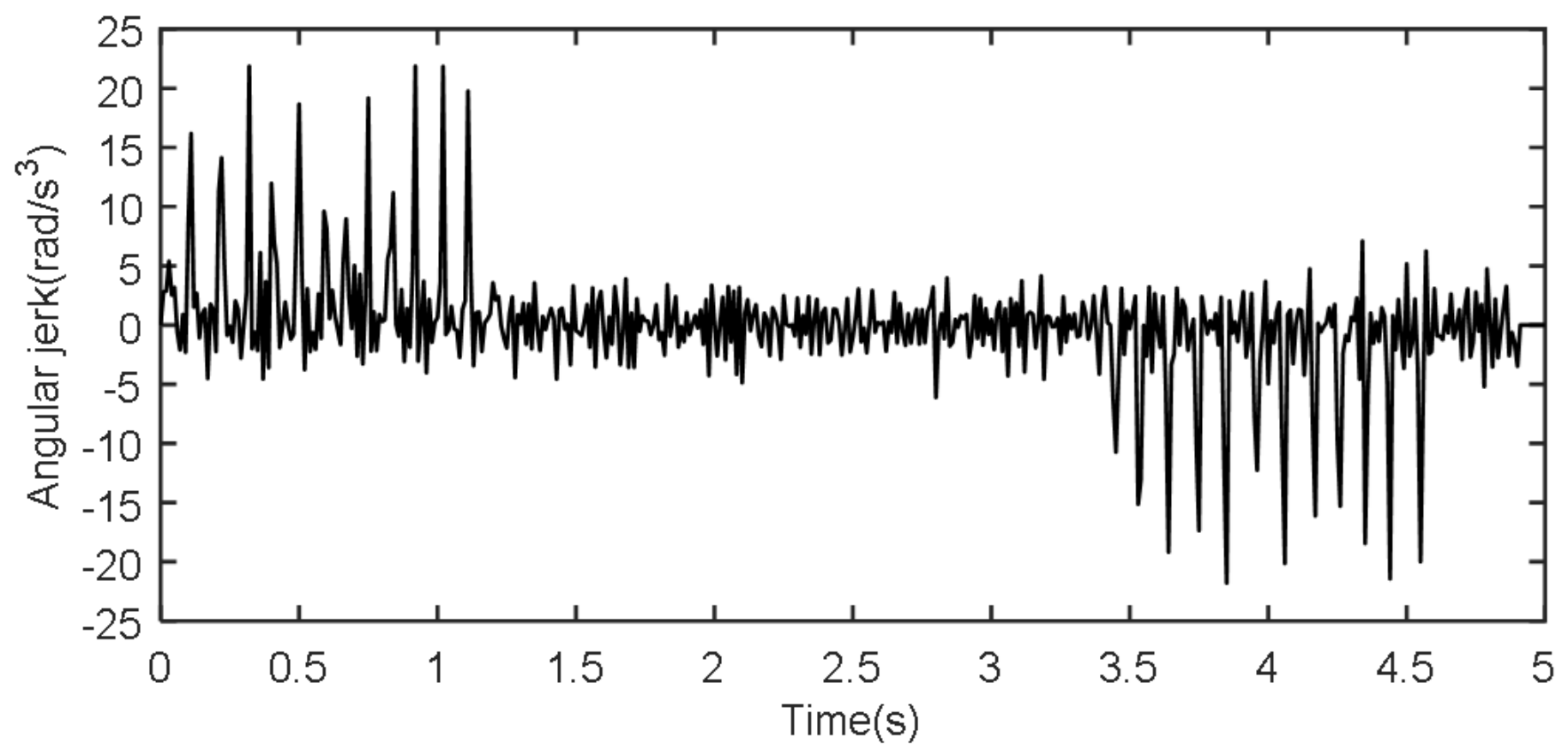

Figure 14

Angular jerk curve based on the modified MFAC method with jerk compensation. 


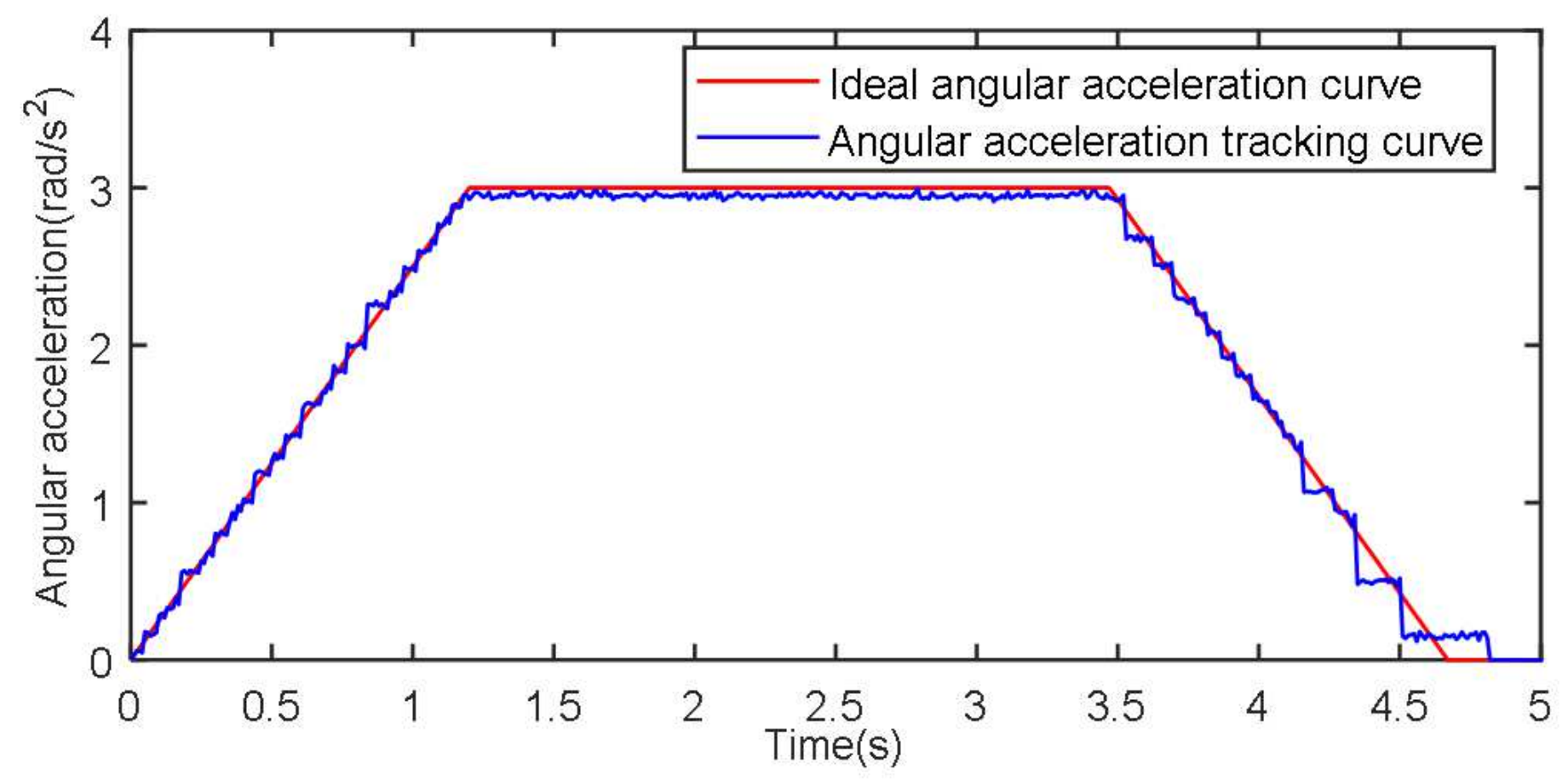

Figure 15

Tracking curve based on the PID method.

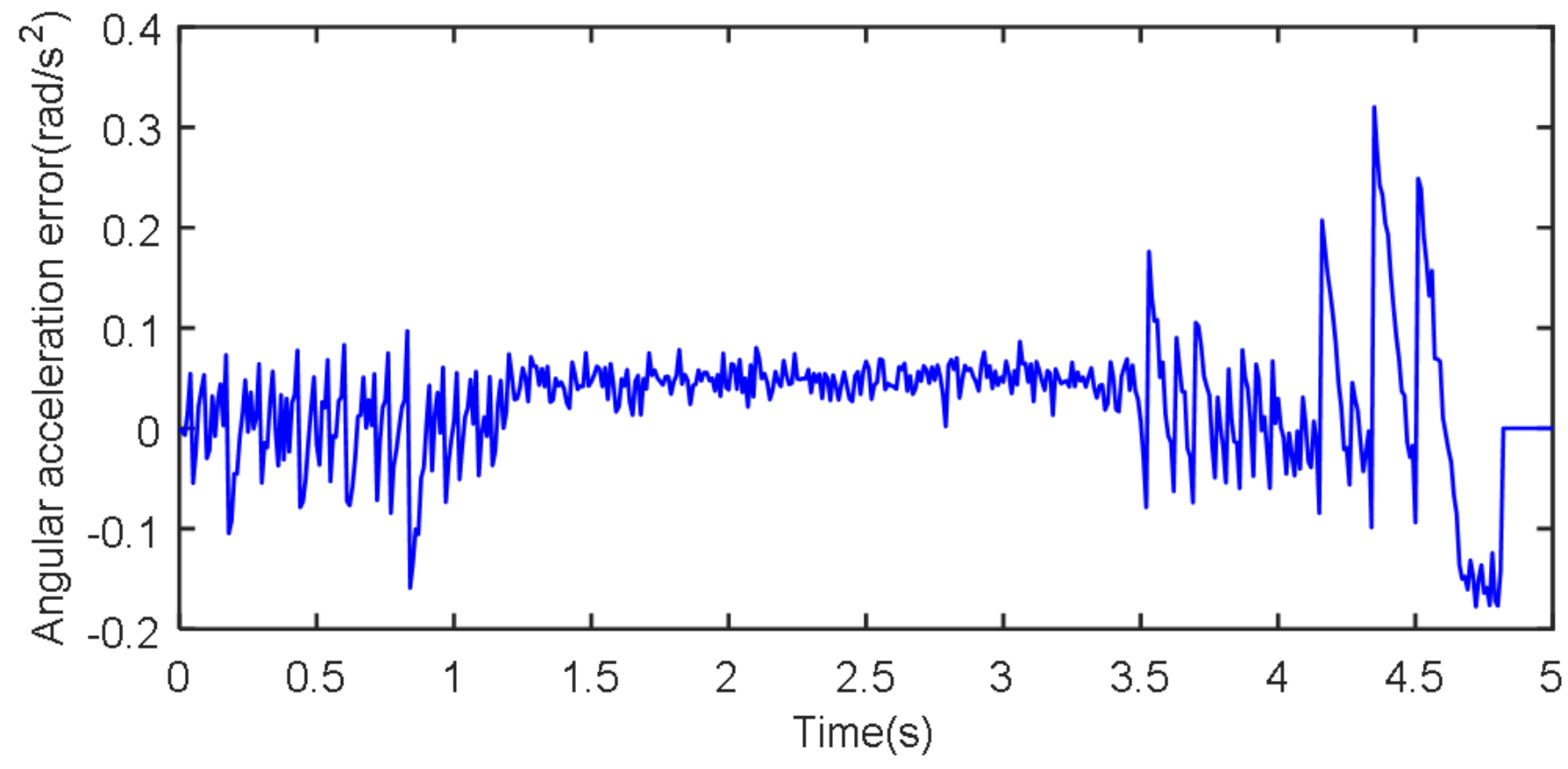

Figure 16

Tracking error curve based on the PID method. 


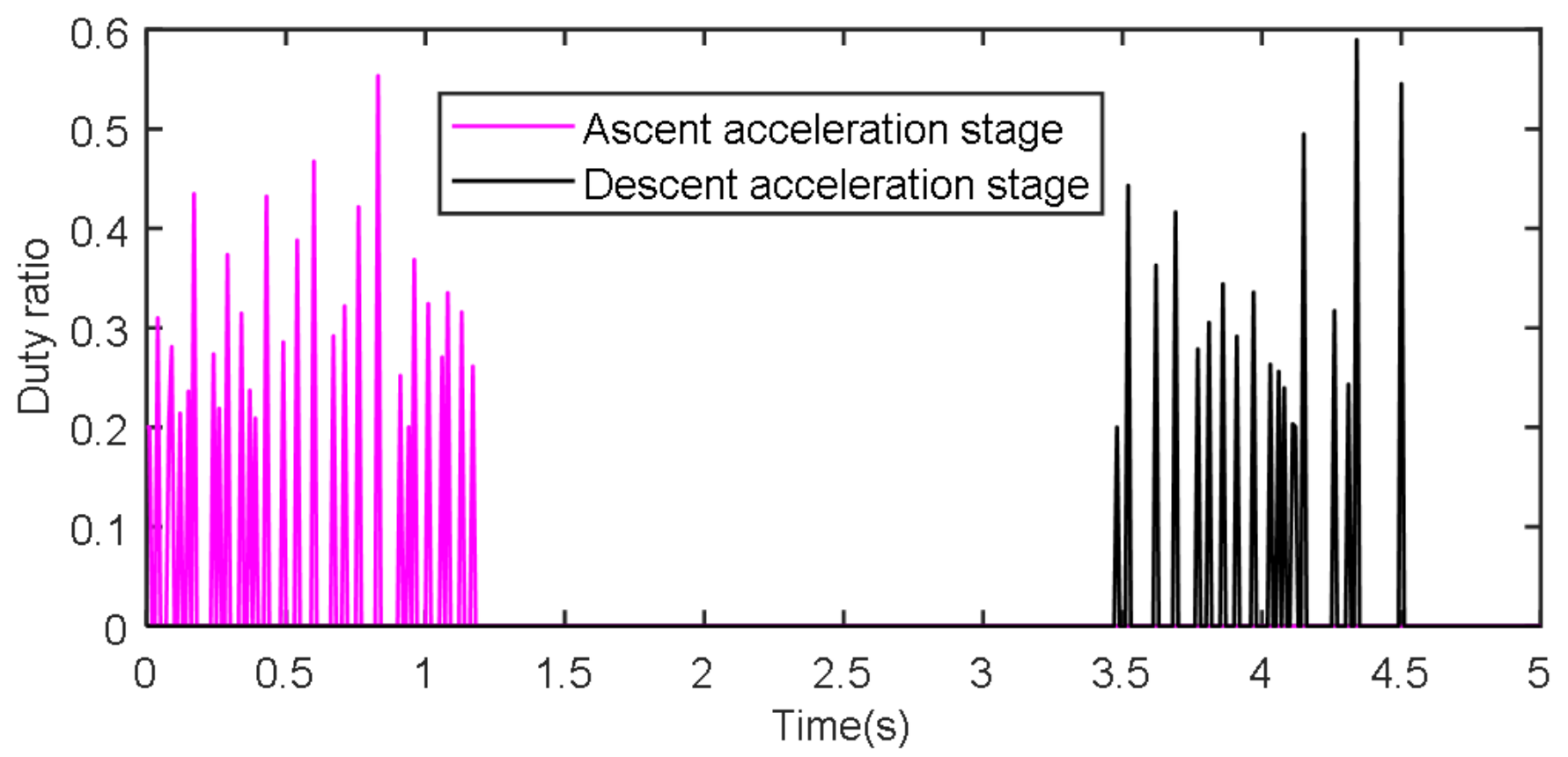

Figure 17

Duty ratio curve based on the PID method.

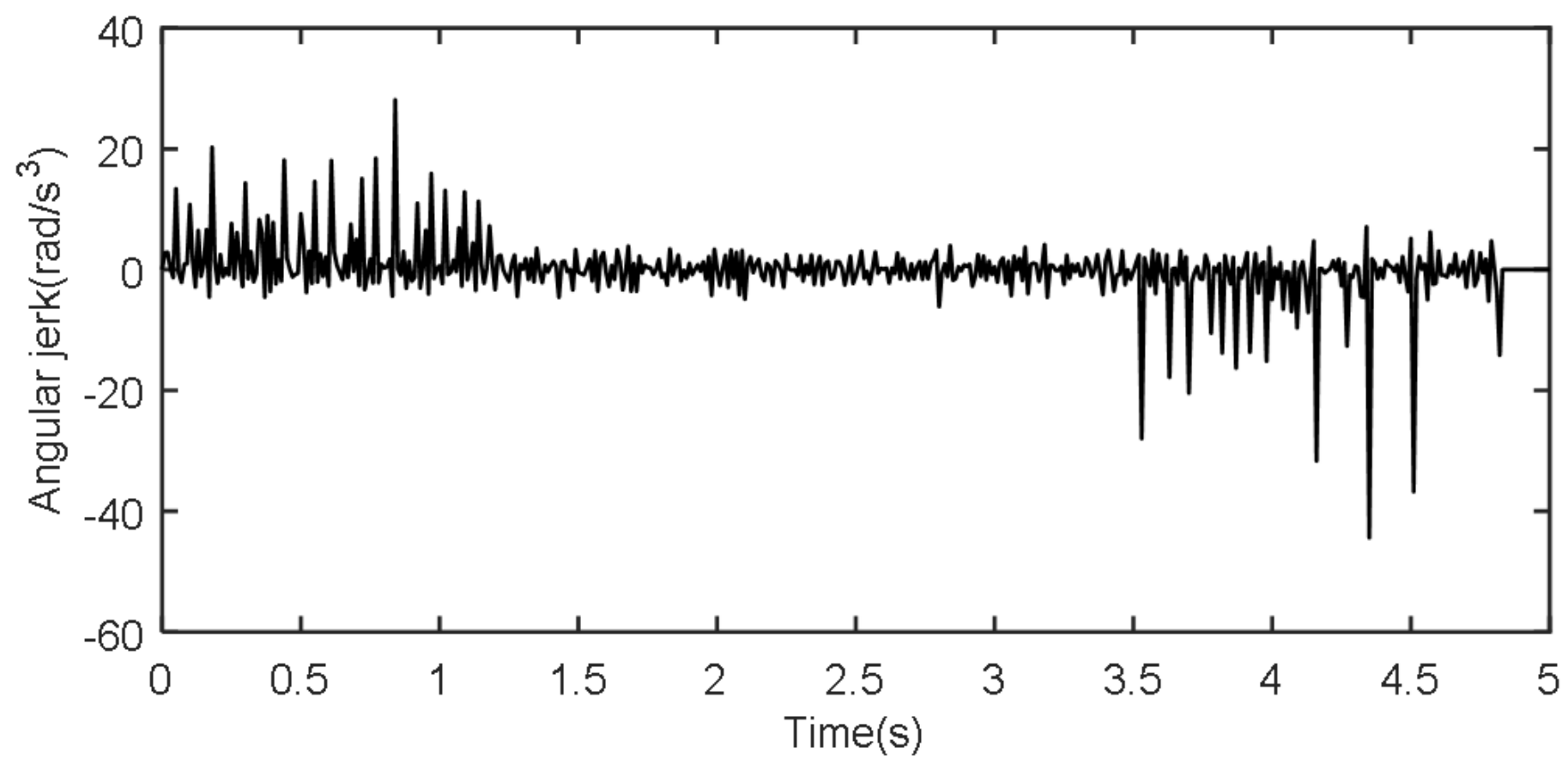

Figure 18

Angular jerk curve based on the PID method. 